"This is a post-peer-review, pre-copyedit version of an article published in Theory and Decision. The final authenticated version is available online at: https://doi.org/10.1007/s11238-016-9573-9"

This version is subjected to Springer Nature terms for reuse that can be found at: https://www.springer.com/gp/openaccess/authors-rights/aam-terms-v1

This item was downloaded from IRIS Università di Bologna (https://cris.unibo.it/)

When citing, please refer to the published version. 


\title{
Pool Size and the Sustainability of Optimal Risk-Sharing Agreements
}

\author{
Francesca Barigozzi ${ }^{a} \quad$ Renaud Bourlès ${ }^{b} \quad$ Dominique Henriet $^{b}$ \\ Giuseppe Pignataro ${ }^{a *}$ \\ ${ }^{a}$ Department of Economics, University of Bologna, P.zza Scaravilli 2, 40126 Bologna, Italy \\ ${ }^{b}$ Centrale Marseille (Aix-Marseille School of Economics), CNRS \& EHESS \\ 38 rue Frédéric Joliot-Curie, 13451 Marseille Cedex 20, France
}

June 2016

\begin{abstract}
We study a risk-sharing agreement where members exert a loss-mitigating action which decreases the amount of reimbursements to be paid in the pool. The action is costly and members tend to free-ride on it. An optimal risk-sharing agreement maximizes the expected utility of a representative member with respect to both the coverage and the (collective) action such that efficiency is restored. We study the sustainability of the optimal agreement as equilibrium in a repeated game with indefinite number of repetitions. When the optimal agreement is not enforceable, the equilibrium with free riding emerges. We identify an interesting trade-off: welfare generated by the optimal risk-sharing agreement increases with the size of the pool, but at the same time the pool size must not be too large for collective choices to be self-enforcing. This generates a discontinuous effect of pool size on welfare.

Keywords: optimal risk-sharing agreement, loss-mitigating actions, repeated interactions, collectively optimal vs Nash behaviors.

Jel Classification: D82, D81.
\end{abstract}

\footnotetext{
*E-mail addresses: francesca.barigozzi@unibo.it; dominique.henriet@centrale-marseille.fr; giuseppe.pignataro@unibo.it
}

renaud.bourles@centrale-marseille.fr; 


\section{Introduction}

"[U]nless the number of individuals in a group is quite small, or unless there is coercion or some other special device to make individuals act in their common interest, rational, self-interested individuals will not act to achieve their common or group interests." (Olson 1965, p. 2)

In line with the previous quotation, we study the emergence of the optimal collective action as a self-enforcing mechanism in organizations promoting mutual help among participants. That is, we investigate how members of risk-sharing agreements can pursue a fully shared objective function (the symmetric Pareto efficient or optimal risk-sharing agreement), instead of maximizing their individual objective functions by following Nash behaviors.

In risk-sharing agreements, members contribute whatever amount is needed to meet the losses covered by the pool (see Picard 2014 and references within). Thus, the contribution to be paid is stochastic because it depends on how many individuals suffer the loss in the relevant period.

In our setting, members of the pool are all identical: they are endowed with the same initial wealth, they face the same risk and they are characterized by the same utility function. This allows us to naturally define the optimal risk-sharing agreement and to focus on the behavior of the representative member 1 Pool members face the risk of a monetary loss whose magnitude is affected by a costly action that we assume to be non contractible. We consider two strategies regarding the choice of the action: members can play their Nash strategy or they can choose the collective action. The latter is the action that maximizes the expected utility of a representative individual.

Moreover, the total amount to be reimbursed is financed with equal shares by members of the pool. Importantly, in the first stage, the "pool" decides the share of the monetary loss to be covered in case of damage (thus the coverage maximizes the expected utility of a representative member) then, in the second stage, each member individually chooses the loss-mitigating action. Thus, members' behavior in the second stage affects the pool's choice of the coverage in the first stage.

In this setting we study under which conditions the optimal collective action emerges as an

\footnotetext{
${ }^{1}$ We refer the reader to Footnote 4 and to the end of Section 8 for a discussion about the consequences of relaxing the assumption of uniform wealth in the pool.
} 
equilibrium and we investigate the impact of pool size on members' expected welfare.

The problem is relevant for many organizations we observe in reality. Besides mutual health insurance that we discuss in details in Section 7 other examples are the following. In reinsurance pools, insurers pool their risks together by sharing claims. Companies in the agreement have an obvious common interest in loss prevention and in the maintenance of quality standards throughout the membership. Families in poor countries are usually exposed to substantial risk but formal insurance is rare so that households mostly rely on informal risk-sharing arrangements. Membership requires trust and only families with a good reputation of being careful and reliable can participate in the pool. Maritime insurance is another example2, Typically, P\&I Clubs offer "protection and indemnity"to shipowners in the form of risk-sharing contracts. In the risk-sharing agreement shipowners are supposed to behave according to precise safety rules. Lawyers, doctors and business consultants highly rely on partnership contracts. The agreement specifies rules on how risk, costs and profits are shared in the partnerships. Partners have a common interest in risk and cost reduction.

In our setting, Nash behaviors allow for a partial internalization of the beneficial effect of the members' action on the required contribution, while the optimal collective action allows for a full internalization and thus improves efficiency and welfare. We first show that full coverage is the pool's optimal choice in case the optimal collective action is pursued, while partial coverage is optimally chosen by the pool under Nash behavior. Moreover, as the size of the pool tends to infinity, the optimal risk-sharing agreement reproduces the first-best while Nash behaviors lead to the same welfare generated by a coinsurance policy with fixed contribution (premium) ${ }^{3}$ Then, we investigate how the efficient action, that is the optimal collective action, can be sustained as an equilibrium in our framework.

As is well known, optimal collective actions can sometimes be obtained with repeated interactions and some punishment strategy in the case of deviation (hence, with respect to the initial quotation from Olson 1965, the "special device" we consider in the paper is repeated interaction).

\footnotetext{
${ }^{2}$ For a brief overview see http://www.igpandi.org/Group+Agreements/The+Pooling+Agreement, consulted in November 2015.

${ }^{3}$ Contracts with fixed premium are typically offered by traditional forprofit companies and correspond to policies where the risk is transferred, in full or in part, to the insurer.
} 
We mainly focus on the "trigger" strategy but we also briefly discuss other punishment strategies. When studying the repetition of the game, we investigate the case of full commitment as for the coverage offered in the agreement in the first stage, meaning that the coverage remains fixed in the case of deviation and punishment (as it seems plausible if the coverage is defined by a contract). However, we show that results are qualitatively equivalent when both stages (the choice of the coverage and the choice of the action) are repeated, so that the pool selects a new coverage after deviation.

We show that the free-riding incentives hampering the optimal risk-sharing agreement are increasing in the size of the pool. Our setting thus allows the identification of a trade-off that has important policy implications because it dramatically affects efficiency in risk-sharing arrangements. On the one hand, efficiency is increasing in the number of participants in the risk-sharing agreement. On the other hand, since members' incentives to free ride are always higher in a large pool, the optimal risk-sharing agreement is enforceable only when the size of the pool is not too high. Therefore, when the dimension of the pool is relatively large, Nash behaviors prevail and lead to the inefficient equilibrium with free-riding. This depicts a schedule for members' expected utility in equilibrium that is discontinuous, with a downward jump when optimality turns out to be no longer enforceable. With the help of simulations performed with CARA and CRRA utility functions, we also show that, except for the size of the pool such that the optimal risk-sharing agreement is no longer enforceable (the downward jump), welfare is increasing in the pool size, even when Nash behaviors emerge. Hence, the efficient size of the pool is either the maximal one compatible with cooperation or the largest possible (see Figure 3).

Our results are in line with some theoretical papers showing that contracts in small pools allow better coordination of members' behaviors (see, for example, Ligon and Thistle, 2005; Fafchamps and Lund, 2003; Genicot and Ray, 2003 or Bramoullé and Kranton, 2007) and with some evidence that we discuss in Section 7 and in the Conclusion.

A relatively large academic literature has analyzed risk-sharing agreements in the last decades. Since Borch (1962), theorists have evaluated different risk-sharing policies comparing them in particular to the policies with fixed premium (see Eeckhoudt and Kimball, 1992; Doherty and Dionne, 
1993). Risk-pooling agreements have been analyzed in settings with asymmetric information by, among others, Mayers and Smith (1986), Smith and Stutzer (1990), Lee and Ligon (2001), Ligon and Thistle (2005) and Bourlès and Henriet (2012). Within this literature, the contribution most closely related to our paper is Lee and Ligon (2001) who study optimal risk-sharing contracts in the case of moral hazard. In particular, their policyholders choose non-cooperatively an action affecting the probability of the loss, while we analyze the choice of an action affecting the size of the loss and we focus on Pareto efficient behaviors as an alternative to Nash behaviors.

The structure of the article is described below.

Section 2 describes the economic environment. The optimal risk-sharing agreement is defined and characterized in Section 3. The risk-sharing agreement with free-riding is characterized in Subsection 4. Subsection 5analyzes the relationship between pool size, action efficiency and members' welfare. Section 6 shows how the optimal risk-sharing agreement can be enforced in the case of repeated interactions. Section 7 discusses mutual health insurance as a real world example fitting with our setting and results. Concluding remarks follow in the last section.

\section{Model set up}

A pool is a set $N$ of $n$ identical individuals whose utility from money is represented by a strictly increasing and strictly concave Von Neumann-Morgenstern utility function $U(\cdot)$, differentiable at least twice with $\lim _{c \rightarrow+\infty} U^{\prime}(c)=0$. Individuals all have initial wealth $w$ and face the probability $p$ of a monetary loss of size $L(a)$ with independently and identically distributed risks $4^{4}$ The loss function depends on a member's action $a \in[0,+\infty)$, it is a positive and twice differentiable function such that $L^{\prime}(a)<0$ and $L^{\prime \prime}(a)>0, \forall a \in[0,+\infty) !^{5}$ The loss-mitigating action is chosen before the risk is realized and implies a disutility cost $C(a)$, with $C(0)=0, C^{\prime}(a) \geq 0$ and $C^{\prime \prime}(a)>0$,

\footnotetext{
${ }^{4}$ The specification of identical wealth for all individuals simplifies the analysis. It allows us to focus on agents with identical risk perceptions. Heterogeneity would call for further assumptions on risk aversion (whether it is increasing or decreasing in wealth), on the collective objective of the pool (how the agreement aggregates utility of different members) and on the negotiation process. In the conclusion we provide a discussion on how relaxing such assumption would affect the analysis.

${ }^{5}$ As an example, suppose that individuals are facing the risk of illness. Prevention is the loss-reducing action implying an ex-ante utility cost and having a benefit in terms of a lower health shock in the case of illness. In Ehrlich and Becker (1972), a consumer's action decreasing the amount of a possible loss is called a self-insurance measure.
} 
$\forall a \in[0,+\infty)$, which is additively separable from the utility derived from money. We furthermore assume that $C^{\prime}(0)=0$.

In the risk-sharing agreement, members pay a contribution and receive a reimbursement if the loss occurs. As usual with this kind of structure, we require the individual action $a$ to be not perfectly controllable. To this end we restrict the set of feasible contracts assuming a simple partial reimbursement $q L$ according to a fraction $q \in[0,1]]^{6}$ This cost-sharing or linear contract indeed represents the type of contract mostly offered by (formal and informal) risk-sharing agreements and standard insurers. Alternatively, to guarantee that the action $a$ cannot be controlled, we could have considered richer contracts (rarely observed in reality), but at the cost of adding a stochastic mapping between $a$ and $L$. This alternative would have significantly obscured the analysis without qualitatively affecting our results (see the discussion at the end of Section 6).

Let $\widetilde{\delta}_{j}, j \in\{1, \ldots, n\}$, be the simple Bernoulli variable, equal to 1 with probability $p$ and 0 with probability $1-p$, i.e. $(1, p ; 0,1-p)$. The total amount of reimbursements to be paid by the pool is thus $q \sum_{j=1}^{n} L\left(a_{j}\right) \widetilde{\delta}_{j}$. We assume that such an amount is equally shared among the $n$ individuals so that each member pays the contribution $\frac{q}{n} \sum_{j=1}^{n} L\left(a_{j}\right) \widetilde{\delta}_{j}$. Thus, in the risk-sharing agreement, the contribution to be paid in order to receive the insurance coverage is random because it depends on the realization of the Bernoulli variable $\widetilde{\delta}_{j}$ for each individual 7

The expected utility achieved by member $i$ is:

$$
u_{i}(\vec{a}, q, n)=\mathbb{E} U\left(w-\left((1-q) L\left(a_{i}\right) \widetilde{\delta}_{i}+\frac{q}{n} \sum_{j=1}^{n} L\left(a_{j}\right) \widetilde{\delta}_{j}\right)\right)-C\left(a_{i}\right)
$$

where $\vec{a}$ stands for the vector $\left(a_{j}\right)_{j=1}^{n}$.

The timing of choices in the pool is the following:

Stage 1 The pool collectively commits to a coverage level $q$.

Stage 2 (i) Each member chooses his/her own action. (ii) The risks are realized. The pool collects

\footnotetext{
${ }^{6}$ Clearly, with a non-linear contract paying $-\infty$ if the loss is higher than the one associated with the desired action, the efficient action would be implementable.

${ }^{7}$ This represents a major difference with respect to the standard insurance policy with fixed premium that we analyze in Appendix 9.5
} 
the contributions and pays reimbursement to those members who suffered the loss.

Importantly, the pool collectively chooses the optimal coverage $q$ and then each member chooses his/her loss-mitigating action $a_{j}$ given the coverage $q$. Notice that since the pool commits to the coverage in Stage 1, only the second stage of the game, i.e. the choice of the action, can be possibly repeated (see Section 4 for the definition of the static game and Section 6 for the description of the repeated game).

Below we first define the optimal risk-sharing agreement, that is the agreement in which all members fully internalize the positive externality their action exerts on the contribution to be paid for membership. As we will explain, the action chosen in the optimal risk-sharing agreement is the optimal collective action, in line with Olson's (1965) idea of an action that represents the common interest of a group (see also Ostrom 2000). In the subsequent section we will analyze the Nash equilibrium arising in the risk-sharing agreement when individuals pursue their own personal interest.

\section{The optimal risk-sharing agreement}

In this section we characterize the optimal risk-sharing agreement under anonymity. The anonymity assumption guarantees that all members perform the same action and that the objective function of the pool is the maximization of the expected utility of a representative agent.

The optimal risk-sharing agreement is defined below.

Definition 1 The optimal risk-sharing agreement maximizes the expected utility of a representative member of the pool with respect to both the coverage $q$ and the collective action a. We denote by $a^{C}$ the resulting optimal collective action 8

As all members choose the same action, the expected utility of a representative member writes:

$$
u_{i}^{C}(a, q, n) \equiv u_{i}([a, \ldots, a], q, n)=\mathbb{E} U\left(w-\left((1-q) \widetilde{\delta}_{i}+\frac{q}{n} \sum_{j=1}^{n} \widetilde{\delta}_{j}\right) L(a)\right)-C(a)
$$

\footnotetext{
${ }^{8}$ In this sense this is also the symmetric Pareto efficient agreement.
} 
where, once again, action $a$ is the same for all members of the pool by construction.

Notice that all individuals fully internalize the positive externality exerted by their action on the total contribution required for membership, $\frac{q}{n} \sum_{j=1}^{n} \widetilde{\delta}_{j} L(a)$. In different words, the loss-mitigating action will be optimally chosen by taking into account its social benefit in terms of a lower contribution to be paid by all members of the pool.

Given Definition 1 above, the optimal collective action $a^{C}(q, n)$ is the solution of:

$$
\mathbb{E}\left[U^{\prime}\left(w-\left((1-q) \widetilde{\delta}_{i}+\frac{q}{n} \sum_{j=1}^{n} \widetilde{\delta}_{j}\right) L(a)\right)\left((1-q) \widetilde{\delta}_{i}+\frac{q}{n} \sum_{j=1}^{n} \widetilde{\delta}_{j}\right)\right]=-\frac{C^{\prime}(a)}{L^{\prime}(a)}
$$

We call $q^{* C}$ the optimal coverage in the optimal risk-sharing agreement. By solving the program where expected utility $(2)$ is maximized with respect to the coverage and where the action satisfies (3), one can show that:

Proposition 1 The optimal risk-sharing agreement offers full coverage $\left(q^{* C}=1\right)$ to its members.

\section{Proof. See Appendix 9.1}

Intuitively, since the collective action fully internalizes the positive externality on the contribution to be paid for membership, full coverage is optimal. In different words, the pool does not need to limit free-riding by imposing partial coverage, as will be instead the case in the case in which individuals play their Nash strategy.

Substituting $q^{* C}=1$ in (3), the optimal action from the point of view of the pool, $a^{C}(1, n)$, is the solution of:

$$
\mathbb{E}\left[U^{\prime}\left(w-L(a) \frac{\sum_{j=1}^{n} \widetilde{\delta}_{j}}{n}\right) \frac{\sum_{j=1}^{n} \widetilde{\delta}_{j}}{n}\right]=-\frac{C^{\prime}(a)}{L^{\prime}(a)}
$$

Note that, despite full coverage, members still face some risk because the contribution to be paid is random and depends on how many losses realize in the pool.

As a last remark we would like to emphasize the similarity between the optimal risk-sharing agreement and the first-best linear policy with fixed contribution presented in Appendix 9.2. In- 
tuitively, both with random and with fixed contribution, the optimal policy entails full-coverage because the optimal action is enforceable.

\section{The risk-sharing agreement with free-riding}

In this section we study the risk-sharing agreement in which members of the pool do not internalize the full benefit of their action on the contribution to be paid for membership, that is they free-ride on the loss-mitigating action. It is important to consider, however, that the pool still chooses the coverage $q$ collectively in Stage 1 .

In Stage 2, pool members play the game, $\Gamma\left(N, \vec{a} \in R_{+}^{n},\left(u_{i}\right)_{i \in\{1 \ldots n\}}\right)$, where $N$ identifies the set of $n$ players and $\vec{a}$ is the vector of pure strategies $\left(a_{i}\right)_{i \in\{1 \ldots n\}}$ with $a_{i} \in[0,+\infty)$.

Below we thus first characterize the Nash equilibria of this game and then we derive the optimal coverage $q$ chosen in Stage 1 when the pool anticipates that members will play Nash equilibria in Stage 2 .

Denoting by $\vec{a}_{-i}=\left(a_{j}\right)_{j \neq i}$ the strategies (actions) of all agents other than $i$, expression 11 can be written as:

$$
u_{i}\left(\left[a_{i}, \vec{a}_{-i}\right], q, n\right)=\mathbb{E} U\left(w-\left((1-q) L\left(a_{i}\right) \widetilde{\delta}_{i}+\frac{q}{n}\left(L\left(a_{i}\right) \widetilde{\delta}_{i}+\sum_{j \neq i} L\left(a_{j}\right) \widetilde{\delta}_{j}\right)\right)\right)-C\left(a_{i}\right)
$$

where, in the contribution of individual $i$, we now have two terms: the first indicates the contribution to be paid in order to cover individual $i^{\prime}$ s possible loss, the second term indicates the contribution required to cover the possible loss of all the other members. Expression (5) shows that each member perceives his/her own action as playing a role only when the negative shock realizes $\left(\delta_{i}=1\right)$.

In Stage 2, i.e. in game $\Gamma$, each member maximizes expected utility 5 taking as given the action chosen by other individuals $\vec{a}_{-i}$ and the coverage $q$.

Let us define $B R_{i}\left(\vec{a}_{-i}\right) \equiv \arg \max _{a_{i}} u_{i}\left(\left[a_{i}, \vec{a}_{-i}\right], q, n\right)$ individual $i$ 's best-response, i.e. the $a_{i}$ 
which solves:

$$
\mathbb{E}\left[U^{\prime}\left(w-\left(\left(1-q+\frac{q}{n}\right) \widetilde{\delta}_{i} L\left(a_{i}\right)+\frac{q}{n} \sum_{j \neq i} \widetilde{\delta}_{j} L\left(a_{j}\right)\right)\right) \widetilde{\delta}_{i}\right]\left(1-q+\frac{q}{n}\right)=-\frac{C^{\prime}\left(a_{i}\right)}{L^{\prime}\left(a_{i}\right)}
$$

Interestingly, the best response $B R_{i}(\cdot)$ would be positive (whatever the actions chosen by other members) even in the case of full coverage $(q=1)$. Indeed even at $q=1$, the left-hand side of $(6)$ is strictly positive, which implies $a_{i}>0$, as $C^{\prime}(0)=0, C^{\prime \prime}(a)>0$ and $L^{\prime}(a)<0$. The reason is that individuals internalize part of the beneficial impact of their action on the contribution through the term $\frac{q}{n} \widetilde{\delta}_{i} L\left(a_{i}\right)$ appearing on the left-hand side of equation $(5)$.

One can then show that a Nash symmetric equilibrium exists and is unique:

Lemma 1 In the risk-sharing agreement with free-riding, a Nash equilibrium in pure strategies exists. It is symmetric and unique and corresponds to $a^{N}(q, n)$ solving in a

$$
\mathbb{E}\left[U^{\prime}\left(w-\left((1-q) \widetilde{\delta}_{i}+\frac{q}{n} \sum_{j=1}^{n} \widetilde{\delta}_{j}\right) L(a)\right) \widetilde{\delta}_{i}\right]\left(1-q+\frac{q}{n}\right)=-\frac{C^{\prime}(a)}{L^{\prime}(a)}
$$

Proof. See Appendix 9.3

We now consider Stage 1. The coverage is chosen collectively by the pool, thus $q^{* N}$ is the solution to the program where the expected utility of the representative individual (5) is maximized under the free-riding constraint $a_{j}=a^{N}(q, n), \forall j=1 \ldots, n$.

Proposition 2 The risk-sharing agreement with free-riding optimally offers partial coverage $\left(q^{* N}<1\right)$ to its members.

Proof. See Appendix 9.4

Here, members of the pool receive a partial reimbursement of their monetary losses ${ }^{9}{ }^{10}$ Intuitively, members need incentives, in the form of partial coverage, because they do not fully internalize

\footnotetext{
${ }^{9}$ From an analytical point of view, full coverage is not optimal anymore in the risk-sharing agreement with freeriding because the Envelope Theorem cannot be applied in this case. See, in particular, equations 14 and 27] in Appendix 9.1 and 9.4 respectively.

${ }^{10}$ Lee and Ligon (2001) analyze a risk-sharing agreement where a non-contractible self-protection action is available
} 
the benefit of their action on the contribution to be paid.

We would like to stress the similarity between the risk-sharing agreement with free-riding and the second-best linear policy with fixed contribution (typically offered by standard insurance companies) that we present in Appendix 9.5 Both with random and with fixed contribution, the policy entails partial coverage because the action cannot be controlled. Notice however that, because of the random contribution, in the risk-sharing agreement with free-riding individuals face a higher risk than the one they face in the case of the second-best policy with fixed premium ${ }^{11}$ We thus expect the partial coverage chosen by the pool to be higher than the second-best partial coverage with fixed contribution.

\section{How pool size affects the risk-sharing agreement}

We now consider how the members' payoff changes with the size of the pool.

We first observe that, as expected, individuals are better off in the optimal risk-sharing agreement than in the one with free-riding. Moreover, as the pool size goes to infinity, we show that the optimal risk-sharing agreement converges to the first-best allocation derived with a linear policy with fixed contribution (see Appendix 9.2 whereas, the risk-sharing agreement with free-riding is equivalent to the second-best policy with fixed contribution (see Appendix 9.5). Finally we show that, in the optimal risk-sharing agreement, members' payoff is increasing in the pool size.

First of all consider that, when $n=1$, the payoffs with and without free-riding are identical and do not depend on the coverage $q$ :

$$
\mathbb{E} U\left(w-\widetilde{\delta}_{1} L(a)\right)-C(a)
$$

Moreover, because of the optimality of the collective action:

to policyholders (i.e. in the case of an action that decreases the probability of the loss). Using "Cournot conjectures" they show that full coverage is optimal. Our result differs from theirs since we solve the problem using the concept of Nash equilibrium. This implies that, when choosing the reimbursement level $q$ in the first stage, individuals anticipate the effect of such a coverage on the choice of other members of the pool.

${ }^{11}$ In fact, under full coverage $(q=1)$, we would observe a positive action in the risk-sharing agreement with freeriding (as mentioned below equation 6), whereas we would observe no-action in the case of a linear policy with fixed contribution. 
Observation 1 Given a pool size strictly larger than 1, expected utility in the optimal risk-sharing agreement is always higher than in the risk-sharing agreement with free-riding.

Now, let's consider how expected utility changes with the pool size in the optimal risk-sharing agreement:

Proposition 3 In the optimal risk-sharing agreement, members' expected utility is monotonically increasing in the pool size.

Proof. See Appendix 9.6.

Unfortunately, the proof of Proposition 3 cannot be easily extended to the risk-sharing agreement with free-riding, because of the partial coverage characterizing the Nash equilibrium. Since the benefits of risk-sharing increase with the size of the pool in the agreement with free-riding as well, we expect the individuals' welfare to be increasing with the size of the pool in the Nash agreement too. Our intuition is confirmed by the following simulations (see also Figure 1 below).

Simulations Let us consider the following CARA utility function 12

$$
U(W)=-\frac{e^{-\rho W}}{\rho}
$$

where $\rho$ represents the degree of constant absolute risk-aversion and is assigned the value of 0.5 . Wealth $w$ is 10 and the probability of loss $p$ is 0.3 . The cost of action is expressed by the function $C(a)=a^{2}$, while the loss function is $L(a)=1-a$. Members' expected utility with and without free-riding is shown in Figure 1 below. First, as stated in Proposition 3 , expected utility in the optimal risk-sharing agreement is monotonically increasing in the size of the pool. Second, in line with Remark 1, expected utility is higher in the optimal than in the risk-sharing agreement with free-riding for every possible value of the pool size. Finally, as we expected, in the agreement with free-riding expected utility is (monotonically) increasing in the size of the pool. Interestingly, such

\footnotetext{
${ }^{12}$ Simulations' files are available upon request.
} 
monotonicity is preserved with CRRA utility functions ${ }^{13}$

Insert FIGURE 1 here

\subsection{Asymptotic results}

By the law of large numbers, as the number of individuals in the pool rises and tends to infinity, the share of them suffering the loss $\left(\sum_{j=1}^{n} \widetilde{\delta}_{j}\right) / n$ tends towards the probability of the loss $p$. This implies that, in a very large pool, the random membership contribution $\sum_{j=1}^{n} \widetilde{\delta}_{j} q L(a) / n$ tends to the fixed contribution $p q L(a)$, thus the uncertainty on the contribution disappears precisely as in the first- and in second-best contracts analyzed in Appendix 9.2 and in Appendix 9.5 , respectively. This occurs irrespective of whether the action is the optimal collective or the Nash action. Further, in the optimal risk-sharing agreement, since full coverage is offered, the fixed contribution becomes $p L(a)$.

Let's consider first the optimal risk-sharing agreement. From (4), as $n \rightarrow \infty$, the optimal collective action $a^{C}$ solves:

$$
-p U^{\prime}(w-p L(a)) L^{\prime}(a)=C^{\prime}(a)
$$

which implicitly defines the first-best action under a fixed contribution (see equation 16 in Appendix 9.2. Therefore, when $n \rightarrow \infty$, the optimal risk-sharing agreement replicates the first-best policy with fixed contribution whose welfare is described by (17).

Let's now consider the risk-sharing agreement with free-riding. As the pool size tends to infinity, the term $\frac{q}{n}$ tends to zero. Therefore, from the first-order condition (7), in the Nash equilibrium the action is the solution of:

$$
-p U^{\prime}(w-(1-q) L(a)-p q L(a))(1-q) L^{\prime}(a)=C^{\prime}(a)
$$

which is equivalent to 29 in Appendix 9.5 Thus, as $n \rightarrow \infty$, the individuals' payoff in the Nash equilibrium is exactly the same as with the second-best policy with a fixed contribution.

\footnotetext{
${ }^{13}$ Simulations with CRRA are available upon request to the authors.
} 
All the previous reasoning is summarized in the following Observation:

Observation 2 When the number of individuals in the pool tends to infinity, the optimal risksharing agreement tends to the first-best policy with a fixed contribution, while the risk-sharing agreement with free-riding tends to the second-best policy with fixed contribution .

Below we verify that, when members interact just once, the optimal collective action is never an equilibrium in the optimal risk-sharing agreement because of the incentives to free ride. We then study conditions such that the optimal collective action can be sustained as an equilibrium in a repeated game where pool members interact an indefinite number of times.

\section{The sustainability of the optimal risk-sharing agreement}

Let's first show why the optimal collective action $a^{C}$ is never an equilibrium in a static framework. Indeed, if coverage is fixed to $q^{* C}=1$ and all other members are choosing the action $a^{C}$, member $i$ has incentive to deviate. In particular, given a pool of size $n$, the best reply action (the action under deviation) satisfies:

$$
a^{D}(n) \equiv \arg \max _{a_{i}} u_{i}\left(\left[a_{i},\left(a^{C}(1, n), \ldots, a^{C}(1, n)\right)\right], 1, n\right)<a^{C}(1, n)
$$

The costly action performed by each individual in the pool exerts a positive externality on the random contribution and the individual prefers to free ride on it. The most advantageous situation for a member of the pool is when all the other individuals internalize the social benefit of the action on the random contribution, while he/she just internalizes the impact of his/her action on his/her own damage in the event of loss. This implies that the optimal collective action $a^{C}(1, n)$ cannot be sustained as an equilibrium if individuals in the pool interact only once ${ }^{14}$

Can the optimal collective action $a^{C}(1, n)$ be sustained as an equilibrium in a repeated game?

As explained in Section 2, we will analyze a situation in which the pool commits to coverage $q$

\footnotetext{
${ }^{14}$ We will study the sustainability of the optimal risk-sharing agreement with respect to individual deviations by members of the pool. Hence we do not study a cooperative game which would instead require the specification of a value function for each possible coalition of agents and the analysis of collective deviations.
} 
in Stage 1, but where Stage 2 is repeated an indefinite number of times. Thus, below, the coverage is fixed at $q^{* C}=1$. This assumption seems the most natural and describes a situation where the pool has commitment power and the policy cannot be subject to frequent changes, typically due to time and administrative constraints 15 At the end of this section, we will however also briefly discuss the case with no commitment on the coverage $q$, i.e. the situation in which the repetition of the game concerns both Stages 1 and 2.

More formally, we study the game in which the Stage 2-game $\Gamma\left(N, \vec{a} \in R_{+}^{n},\left(u_{i}\right)_{i \in\{1 \ldots n\}}\right)$ is indefinitely repeated. The repeated game has the following characteristics.

Discount factor: the parameter $\sigma$ indicates the constant "probability-adjusted" discount factor. Denoting $\Delta$ the constant and common discount factor and $\pi$ the common belief that members have regarding the probability that they will continue to interact from period to periog the probability-adjusted discount factor writes: $\sigma=\pi \Delta$, with $0<\sigma<\pi, \Delta \leq 1 \longdiv { 1 7 }$ Hence, the expected discounted payoff of a member of the pool can be written as: $\sum_{t=1}^{\infty} \sigma^{t-1} u_{i}\left(\vec{a}_{t}, 1, n\right)$, where $\vec{a}_{t}$ is the vector of actions played at time $t$.

Punishment strategy: we assume that members of the pool follow the Grim trigger strategy: in the first period, $t=0$, the individual chooses the optimal action level $a^{C}(1, n)$; in $t \geq 1$ he/she chooses $a^{C}(1, n)$ if in each previous period any losses that have occurred are of size $L\left(a^{C}(1, n)\right)$, otherwise he/she chooses $a=a^{N}(1, n)$ forever 18

Monitoring structure: we assume that individuals are informed about the number, denoted by $k$, of pool members who suffer the loss in each period. Each pool member is required to contribute an amount that is equal to $1 / n$ of the sum of reimbursements to be paid in the pool in each period. In the case one member deviates from the optimal collective action and his/her loss realizes, then all members are required to pay a contribution which is larger than $\sum_{j=1}^{n} \widetilde{\delta}_{j} L\left(a^{C}\right) / n=k L\left(a^{C}\right) / n$

\footnotetext{
${ }^{15}$ Note that, by focusing on full commitment on the policy, our model is mostly suited to describe formal risksharing agreements. See Section 7 for a real world example.

${ }^{16}$ In this sense the number of periods over which the game is repeated is indefinite (see Roth and Murnighan 1978).

${ }^{17}$ See Neyman (1999) for a model in which uncertainty on the duration of the game is interpreted as a small departure from the common knowledge assumption on the number of repetitions.

${ }^{18}$ Such a Grim trigger strategy perfectly fits a non-competitive environment, where there is no choice for individuals except that of staying in the pool. We refer the reader to the end of this section for a discussion about exclusion as a punishment strategy, and about punishment when an outside option exists, for example because profit and nonprofit organizations compete in the market.
} 
because the loss of the deviator is $L\left(a^{D}\right)>L\left(a^{C}\right)$. Hence, a deviation from the optimal collective action is detected only if the deviator experiences the loss, that is with probability $p$. This means that we are in a stochastic environment regarding deviation observability. (See the last part of this section about enlarging the stochastic environment to the loss function) ${ }^{19}$

For expositional reasons, let's call $u^{C}(n)=u_{i}\left(a^{C}(1, n), 1, n\right)$ the individual's (one-period) expected utility in the optimal risk-sharing agreement and $u^{D}(n)=u_{i}\left(\left[a^{D}(n),\left(a^{C}(1, n), \ldots, a^{C}(1, n)\right)\right], 1, n\right)$ his/her (one-period) expected utility when he/she deviates. In the period after deviation, the individual will be detected only if the loss has realized, that is with probability $p$. In that case, all the other members of the pool will react by choosing the Nash action level, with $q=1$ or $a^{N}(1, n)$. Let's call $u^{P}(n)=u_{i}\left(\left[a^{N}(1, n),\left(a^{N}(1, n), \ldots, a^{N}(1, n)\right)\right], 1, n\right)$ the expected utility the deviator obtains when he/she is detected and thus punished. The strategy $a=a^{N}(1, n)$ for the deviator is just the subgame perfect Grim trigger strategy in our game.

With probability $(1-p)$ the deviation is not detected and the deviator obtains the payoff $u^{D}(n)$ also in the period after the deviation. The previous reasoning is repeated in the subsequent periods. After a deviation the individual's payoff can be written as follows:

$$
\begin{aligned}
& u^{D}(n)+\sigma p \frac{1}{1-\sigma} u^{P}(n)+\sigma(1-p)\left\{u^{D}(n)+\sigma p \frac{1}{1-\sigma} u^{P}(n)+\right. \\
& \left.\sigma(1-p)\left[u^{D}(n)+\sigma p \frac{1}{1-\sigma} u^{P}(n)+\sigma(1-p)[\ldots]\right]\right\}
\end{aligned}
$$

Thus, the discounted payoff in the case of deviation is:

$$
\frac{\sigma p}{1-\sigma} \sum_{t=0}^{\infty}(\sigma(1-p))^{t} u^{P}(n)+\sum_{t=0}^{\infty}(\sigma(1-p))^{t} u^{D}(n)
$$

or:

$$
\frac{1}{1-\sigma(1-p)}\left[u^{D}(n)+\frac{p \sigma}{1-\sigma} u^{P}(n)\right]
$$

\footnotetext{
${ }^{19}$ With respect to the setting analyzed in Friedman (1971), our model has two specific features: $(i)$ players do not directly observe the action of other players but they infer deviation through the contribution required for membership and from their knowledge of the number of claims; (ii) detection only occurs if the loss of the deviator realizes, that is with probability $p$.
} 
whereas if the optimal collective action $a^{C}(1, n)$ is chosen forever, the discounted payoff a member obtains is:

$$
\sum_{t=0}^{\infty} \sigma^{t} u^{C}(n)=\frac{1}{1-\sigma} u^{C}(n) .
$$

The optimal collective action can thus be sustained as an equilibrium if the discounted payoff in the optimal risk-sharing agreement dominates the discounted payoff from deviation, or when the inequality below holds:

$$
\frac{1}{1-\sigma} u^{C}(n) \geq \frac{1}{1-\sigma(1-p)}\left[u^{D}(n)+\frac{p \sigma}{1-\sigma} u^{P}(n)\right]
$$

From inequality 10 we can state the following:

Proposition 4 Let $\hat{n}$ be the largest $n$ such that (10) holds with equality. The optimal risk-sharing agreement cannot be sustained as equilibrium for $n>\hat{n}$.

Proof. See Appendix 9.7 .

The left and the right-hand side of 10 are equal for $n=1$ (see the proof in Appendix 9.7); hence $\hat{n} \geq 1$ exists. However, the right-hand side of 10 is larger than the left-hand side for $n=+\infty$. As a consequence, the optimal risk-sharing agreement can be sustained in equilibrium if the left-hand side of the inequality increases faster with the pool size than the right-hand side. In other words, the optimal collective action $a^{C}(1, n)$ is enforceable only if the benefits from risk-sharing for low size pools are larger in the optimal risk-sharing agreement than in the one with free-riding or in the deviation.

The previous proposition states that only a pool size which is not too large is compatible with the optimal risk-sharing agreement. Intuitively, incentives to free ride are higher in a larger pool. In fact, when $n$ is high, $(i)$ by deviating from the optimal collective action, $a^{C}(1, n)$, the individual significantly decreases the cost of his action, whereas deviation has almost no negative effect on the contribution and (ii) the punishment is less costly since the benefit from risk-sharing remains important.

However, it can be $\hat{n}=1$. In such a case, the optimal risk-sharing agreement cannot be sustained. 
As expected, the existence of a pool size strictly larger than 1 that satisfies 10 with equality (i.e. $\hat{n}>1$ ) is more likely the higher the individuals' probability-adjusted discount factor $\sigma$.

As explained in the proof, since we cannot exclude that the left-hand side and the right-hand side of 10 cross each other more than once, $n \leq \hat{n}$ is a necessary but not sufficient condition for the sustainability of the optimal risk-sharing agreement. In particular, a closed subset of $n$ below the threshold $\hat{n}$ may exist, for which the payoff under deviation already prevails. However, in all our simulations with CARA and CRRA, the two sides of 10 ) cross just once for $n>1$ (see Figure 2 below).

To sum up, the model shows that a trade-off arises with respect to the size of the pool. On the one hand, the benefit from risk-sharing is increasing in the number of participants; on the other hand the optimal collective action, and thus efficiency, is only compatible with a pool size that is not too large.

Let's now consider the decision of the pool in Stage 1. Given the values of the parameters $n, \sigma$ and $p$ characterizing the economic environment, the pool chooses the coverage $q$ by anticipating the subsequent repetition of Stage 2 of the game for an uncertain number of periods. In different words, the pool anticipates whether, given the size of the pool $n$, the optimal risk-sharing agreement is self-enforcing or if instead the Nash equilibrium of the one-shot game prevails.

In the subsection below we relate the type of risk-sharing agreement prevailing in equilibrium (and its efficiency) to the size of the pool. Before doing so, we study inequality (10) and we show that the optimal risk-sharing agreement is indeed sustained as an equilibrium in the simulations. We also offer some comparative statics about individuals' risk aversion and about the risk of incurring the loss.

\subsection{Simulations}

In Figure 2 below we depict the left-hand side and the right-hand side of inequality (10) using the utility function and the parameter values considered before, together with a probability-adjusted 
discount factor $\sigma=0.320$ Simulations show that the optimal risk-sharing is sustained for $n \leq$ $\hat{n}=1000$. For a larger pool size, the Nash equilibrium emerges and the risk-sharing agreement is not efficient anymore. Note also that, in the example considered, the right-hand side of $(10)$ is monotonically increasing in the pool size. Moreover, the left- and the right-hand side of 10 cross just once for $n>1$. We also obtain monotonicity of the right-hand side and single-crossing with the simulations based on a CRRA function that are available upon request to the authors.

Result 1 (simulations with CARA and CRRA utility functions) The right-hand side of (10) crosses the left-hand side from below and the optimal risk-sharing agreement is self-enforcing if and only if $n \leq \hat{n}$.

\section{Insert FIGURE 2 here}

As basic comparative statics, we can take into account the effects of changing two crucial variables in the analysis of inequality (10): the risk aversion (captured by the parameter $\rho$ ) and the probability of loss $(p)$, respectively. It is worth noting that an increase in the individuals' risk aversion from $\rho=0.5$ to $\rho=0.6$ leads to a large rise in the value of the pool size compatible with the collective action from around 1000 to 3000 . This result relies on the fact that, when risk-aversion increases, the benefit of risk-sharing among members in the pool is higher and the utility generated by the collective action grows. Conversely, after a decrease in risk aversion from $\rho=0.5$ to $\rho=0.4$, we observe that the maximum pool size for which the optimal risk-sharing agreement is sustainable is lower than 350. Let's now consider the loss probability $p$. If it increases from $p=0.3$ to $p=0.6$, we observe that the collective action is compatible with a smaller pool size: the upper bound value of the pool size changes from 1000 to 420. At first sight, this result may seem counterintuitive because the probability of the loss corresponds to the probability of detection in our context and, thus, is directly related to punishment enforcement. Such result relies instead on the effect the probability of the loss has on the individuals' optimal action level. Indeed, an increase in the probability of the loss reduces the difference between the collective and the Nash action (as it increases

\footnotetext{
${ }^{20}$ In Figure 2, the graphs showing the left and the right-hand side of 10 are not represented for the pool size $n=1$ because of the scale of the picture.
} 
the benefit from exerting the action) and thus decreases the differences between expected utilities. Symmetrically, reducing the probability from $p=0.3$ to $p=0.2$ increases the maximum size of the pool compatible with the optimal agreement from 1000 to 1600. In a nutshell:

Result 2 (simulations with CARA utility functions) The optimal risk-sharing agreement is sustainable for a larger pool as the probability of the loss decreases and individuals' risk aversion increases.

Figure 3 describes a representative individual's expected utility as a function of the pool size and the feasible equilibrium for the same numerical example we considered above. Except for $n=\hat{n}$, expected utility is increasing in the pool size because the benefits from risk-sharing increase with the size of the pool. In $n=\hat{n}$ we observe a discontinuity, more specifically a downward jump, because the optimal collective action can no longer be sustained and, for $n=\hat{n}$, the Nash equilibrium is reached instead. In terms of the optimal size of the pool, simulations show that it is convenient to have either a pool size equal to $\hat{n}$, so that individuals can benefit from the optimal risk-sharing agreement and the most efficient loss-mitigating action, or a very large size, so that members can benefit from risk-sharing. The previous discontinuity result is summarized as follows.

Result 3 (simulations with CARA and CRRA utility functions) Except for the downward jump for $n=\hat{n}$, expected utility is increasing in the pool size. The optimal size of the pool is either $\hat{n}$ or the largest possible.

Insert FIGURE 3 here

\subsection{Alternative structures of the repeated game}

Commitment. Let's now consider the setting where commitment is limited and the coverage $q$ can be renegotiated. Renegotiation implies that both Stage 1 and Stage 2 of the game are repeated so that, after the detection of a deviation, the pool makes a new decision about coverage $q$. In such a case, the equilibrium of the repeated game is the equilibrium of the one-shot game (with stages 1 and 2) or the Nash equilibrium described in Subsection 4. Thus, the deviation choice is still 
$a^{D}(n)$ but the coverage offered by the pool after deviation is $q^{* N}<1$. Therefore, the punishment after deviation is no longer $u^{P}(n)$ considered above but corresponds to members' utility in the Nash equilibrium. Our main result does not change qualitatively, in the sense that Proposition 4 still holds, but we obviously find a slightly different maximum size of the pool compatible with the optimal risk-sharing agreement. Interestingly, by performing simulations in the case of limited commitment, we observe that $\hat{n}$ is now lower than in Figure 3 (in particular, now $\hat{n}=950$ ). The reason is that, with limited commitment, the punishment utility is $u\left(a^{N}, q^{* N}, n\right)>u^{P}(n)$. Indeed the former punishment utility is computed considering the coverage that is optimal given the noncooperative choice of the effort, while the latter is computed using $q^{* C}=1$ which is suboptimal when the action is $a^{N}$. As a consequence, with limited commitment, inequality 10 is more difficult to be satisfied.

Punishment in a competitive environment. Suppose that other risk-sharing agreements exist or that forprofit insurers are active in the market. We must then explicitly consider these outside options in the punishment strategy that probably becomes less effective. In the interesting case of an environment where our risk-sharing agreement competes with forprofit companies that act strategically, the outside option becomes endogenous and results largely depend on the kind of dynamic contracts offered by the competitors (see for example Moreno et al., 2006 on bonus-malus schemes used in car insurance).

Detection of deviation. In our framework a deviation from the optimal collective action is inferred when members are asked to pay a contribution larger than expected, i.e. when the individual who is deviating suffers the loss. As the literature on firms' collusion shows, it is possible to obtain cooperation also in settings where detection of cheating behaviors is more difficult than in our model (see Green and Porter 198421. In our framework too, but under more stringent conditions, the optimal collective action would still be sustained if the detection of possible deviations were more difficult to infer, as with a stochastic loss function.

\footnotetext{
${ }^{21}$ Green and Porter (1984) examine the nature of cartel self-enforcement in the presence of demand uncertainty. In particular, in their setting, demand fluctuations (that are not directly observed by firms) make the detection of deviation difficult to infer. The collusive equilibria are then less likely and unstable industry performances can occur. Reversionary episodes, where price cut is performed by all firms in the cartel as a punishment strategy, can sometimes happen with no firm really defecting, simply because of low demand.
} 
Less "demanding" coordination. Even though for $n>\hat{n}$ the optimal risk-sharing agreement is not self-enforceable (with the harshest punishment), still the pool may collectively agree on a lower $a^{C}$, thus reducing the temptation to deviate. Considering these less "demanding" collective equilibria would identify intermediate outcomes in between the optimal risk-sharing agreement and the agreement where the Nash action is implemented. Agreeing on a lower $a^{C}$ for $n>\hat{n}$ would still allow for some efficiency gain and thus reduce the downward jump illustrated in Figure 3. However, this would not qualitatively alter our results.

Exclusion. Suppose now that the identity of the deviator is observable, which is much more demanding in terms of information than what we assume in the model 22 Excluding the deviator from the pool is then a possible punishment strategy. Exclusion represents an effective punishment if no other risk-sharing agreement exists and if coverage cannot be purchased in the insurance market, so that the deviator who is excluded from the pool obtains forever his expected utility when not insured: $E U\left(w-\widetilde{\delta}_{1} L(a)\right)-C(a)$. Such a punishment utility would enter condition 10 in place of $u^{P}(n)$ and Proposition 4 would still hold. Interestingly, since $u^{P}(n)$ is increasing in $n$, $u^{P}(n)$ is larger than $E U\left(w-\widetilde{\delta}_{1} L(a)\right)-C(a)$ for $n$ sufficiently high. Hence, if $n$ is sufficiently high, then exclusion turns out to be a more efficient punishment strategy and inequality 10 is more likely to be satisfied 23

\section{A real example: mutual health insurance}

Mutual health insurance is a real-world example that is perfectly coherent with our analysis of collective actions in risk-sharing agreement.

In the case of health insurance, policyholders decide how much to invest in costly preventive measures. In particular, the action in our model corresponds to secondary prevention, or to a costly measure that, in case of illness, reduces health care expenditures necessary to recover (screening procedures allowing for early detection of disease). Previous literature (Barigozzi, 2004, Ellis and

\footnotetext{
${ }^{22}$ This is possible, for example, if the pool reveals to its members the list of individuals entitled for reimbursement, together with the specific amount paid to each of them.

${ }^{23}$ Notice that, after the exclusion of the deviator, the pool will be composed by $n-1$ members, so that sustainability of the optimal agreement will be more likely.
} 
Manning 2007) showed that a copayment on expenditures for preventive care is necessary to encourage preventative activities since, with fixed premium contracts, individuals do not internalize the positive impact of their prevention on the insurance contribution. Instead, by asking for a random premium, mutual insurance allows for partial or full internalization of the positive impact of prevention on the individual's contribution, as we showed in Sections 4 and 6 .

In the health insurance market, an important difference between the marketing strategy of mutual organizations and that of forprofit insurers offering fixed premium policies is that forprofit insurers do not encourage long run enrollment. In fact, fixed premium policies are strictly annual and their renewal is conditional on the individual's health status and age $24{ }^{24}$

From a normative perspective, in order to compare the efficiency of risk-sharing contracts and fixed premium contracts we should take into account the best strategies that forprofit insurers could adopt if long-term contracts were offered. In the previous Section, we briefly discussed how to investigate a setting with risk-sharing arrangements and forprofit companies coexisting in the insurance market (see "Punishment in a competitive environment"). However, let's take for a moment a positive perspective. Since forprofit health insurers do not offer long-term contracts in the real world and do not generally use information on past behaviors to incentivate prevention, our results suggest that contracts offered by mutuals may be more effective in encouraging prevention than fixed premium policies. To see this, suppose that the optimal risk-sharing agreement can be sustained as equilibrium, as Figure 2 shows, for a pool size lower than 1000. For the same functions and parameter values used in the simulations of Section 6, we can evaluate expected utility derived from the second-best contract with fixed premium (see Appendix 9.5. Note that expected utility generated by a second-best policy with fixed premium is independent, by construction, of the pool size; whereas expected utility generated by the optimal risk-sharing agreement is increasing in the pool size, as Proposition 3 states. Simulations show that the optimal risk-sharing agreement dominates the second-best contract with fixed premium for a pool size larger than 250 (see Figure

\footnotetext{
${ }^{24}$ One of the objectives of the Affordable Care Act, recently approved in the USA, is to protect policyholders from the insurers' practice of refusing policy renewal in case of serious health conditions.

${ }^{25}$ Interestingly, long-term contracts are instead offered by standard companies in other insurance markets. For example front-loaded contracts in life insurance generate a partial lock-in of consumers: contracts that are more front-loaded have a lower present value of premiums over the period of coverage (see Hendel and Lizzeri 2003).
} 
4) ${ }^{26}$ This implies that, in this example, a large range of pool sizes exists such that the optimal risksharing agreement is sustained as an equilibrium and the risk-sharing agreement is more efficient than the second-best contract.

\section{Insert FIGURE 4 here}

Our model shows that members of risk-sharing arrangements may find it convenient to choose the optimal collective action and fully cope with it. Indeed, when individuals enroll for a sufficiently high amount of time and do not discount the future too much, the optimal risk-sharing agreement becomes self-enforcing and the largest size of the pool compatible with efficiency results from the trade-off between mutualization and free-riding issues. Interestingly, some evidence confirms the relatively small dimension of mutual companies, especially in Europe. For example, according to the International Cooperative and Mutual Federation (ICMIF) ${ }^{27}$ the total market share of the 2,900 mutual firms active in 75 countries at the end of 2010 was just $26 \%$. More detailed evidence can be found in France and Italy. Caire (2009) reports that, in 2007 in France, the 808 existing mutuals accounted for $58 \%$ of the health insurance market, the 9 active stock (i.e. standard) insurers owned $23 \%$ of the market, while the remaining $19 \%$ was the share of "institutions de prévoyance" (non-profit organizations that offer collective insurance contracts for firms) ${ }^{28}$ Likewise in Italy, health mutual firms are definitely characterized by small size. About 1,500 "Società di mutuo soccorso" (mutual benefit societies) were active in 2010, they had less than one million members and represented around $12 \%$ of the Italian market in complementary health insurance (see Lippi Bruni et al. 2012).

We briefly discuss some other examples in the Conclusion.

\footnotetext{
${ }^{26}$ These simulations are available upon request to the authors.

${ }^{27}$ See the Global Mutual Market Share report 2010, available at http://www.icmif.org/mms2010.

${ }^{28}$ Similarly, Kerleau (2009) shows that the market for mutual contracts is characterized by low concentration in France, as the 5 biggest mutuals in 2005 represented only $20 \%$ of the market share and the 30 biggest ones only $44 \%$.
} 


\section{Concluding remarks}

We have considered an agreement in which individuals share their risk of a monetary loss but have incentives to free ride when choosing a costly loss-mitigating action. We have shown that the optimal collective action can emerge as a self-enforcing mechanism.

The optimal collective action allows individuals to fully internalize the benefit of their action on the contribution to be paid for participating in the agreement. We emphasize the trade-off generated by the fact that both efficiency from risk-sharing and incentives to free ride are increasing in the size of the pool. Since the implementation of the optimal risk-sharing agreement is possible only when the size of the pool is not too high, the benefits from the optimal collective action comes at the cost of limiting efficiency from risk-sharing.

The sustainability of the optimal collective action as equilibrium is consistent with the general view that small pools allow for better monitoring of individuals' behaviors and with the empirical evidence that organizations offering risk-sharing contracts are typically small. Among the motivating examples listed in the Introduction, we mentioned maritime insurance. Interestingly, the number of shipowners in each risk-sharing agreement (Club) is, even on a worldwide basis, fairly limited. The diseconomies of scale arising for Clubs with size above a particular level are typically interpreted as a consequence of increasing coordination costs (see Aase, 2007). Moreover, the fact that only shipowners with a solid reputation of cautious behaviors are admitted to the Club and that Club members incurring reckless or avoidable losses are asked to leave, suggests the enforcement of some kind of collective behavior in the pool. In the same way, the maintenance of high quality standards in reinsurance pools might be interpreted as a sign of cooperation among members of the agreement. In the case of informal insurance in rural villages, the agreement is self-enforcing because failure to make a promised payment translates into a social stigma and the risk of losing membership. Exclusion is costly because individuals derive high utility from participating in the agreement, also in the form of respect and consideration from other people in the village. This is coherent with some form of collective actions being sustained among members of the informal agreement. 
In the model, acting in the interest of the pool does not require an individual to be empathetic with other members, but originates from an absolutely standard utility maximizer attitude. However, supportive, fair and conditional cooperative behaviors can be considered plausible in a risk-sharing agreement, given the very specific nature of the participating contract. In other words, willingness to exert actions that are optimal from the point of view of the organization may be higher for individuals who self-select into organizations selling these contracts. For example, solidarity principles explicitly mentioned in all articles of association/incorporation, if equally shared by agents, could well facilitate collective actions ${ }^{29}$ Moreover, cheating behaviors can also entail some psychological costs to the deviator in terms of, for example, lower self-esteem or a social stigma as in the case of informal insurance. Obviously, some kind of prosocial attitude or altruism would reinforce the sustainability of efficiency (see Alger and Weibull, 2010, on the impact of altruism on risk-sharing). Finally, although remaining in a framework where individuals are characterized by purely selfish preferences, members of the pool partially know each other since they assemble for periodical meetings. As in the case of law partnerships, this suggests that some partial peer monitoring is possible when the pool is not too large, making it easier to sustain equilibria enforcing collective actions.

As a last remark, in our analysis we considered individuals endowed with the same wealth. The analysis of an agreement with identical individuals seems a first natural step in the study of the enforceability of optimal risk-sharing arrangements. It allowed us to compare optimal symmetric choices in the case of risk-sharing agreements with and without free-riding and to offer clear and neat conditions on the pool size for the symmetric Pareto efficient agreement to be self-enforcing. However, relaxing the assumption of identical pool members represents an interesting further step. Suppose for example that two different types of individuals exist, the rich and the poor. Their different wealth is likely to translate into a different attitude toward risk and into different optimal actions; we expect, in particular, rich members to be willing to exert lower loss-mitigating actions than poor members. A specific welfare function for the pool, aggregating the expected utility of

\footnotetext{
${ }^{29}$ The beneficial matching between agents characterized by a similar "mission" (or social attitude) has been analyzed by Besley and Ghatack (2005). They show that fewer incentives are required if employer and employee share the same mission.
} 
rich and poor members, has to be defined together with a specific rule for sharing the cost of the total amount of reimbursement to be paid in the pool. Importantly, if the members' wealth is their private information, then a screening mechanism has to be designed to prevent mimicking in members' actions. In fact, reasonably, under asymmetric information poor members will be tempted to exert less than optimal actions pretending to be rich. According to the sharing rule that is in place in the pool, we expect both separating and pooling equilibria in actions to emerge in the agreement. An asymmetric and constrained efficient risk-sharing agreement would be possibly enforced in such a case.

\section{Appendix}

\subsection{Proof of Proposition 1}

Denote $\widetilde{W}_{i}(\vec{a}, q, n)$ the (stochastic) wealth of individual $i$ under a risk-sharing agreement characterized by $n$ members choosing actions $\vec{a}$, with coverage level $q$ :

$$
\widetilde{W}_{i}(\vec{a}, q, n) \equiv\left((1-q)\left(w-\tilde{\delta}_{i} L\left(a_{i}\right)\right)+q\left(w-\frac{\sum_{j=1}^{n} \tilde{\delta}_{j} L\left(a_{j}\right)}{n}\right)\right)
$$

Importantly, the second term $q\left(w-\frac{\sum_{j=1}^{n} \tilde{\delta}_{j} L\left(a_{j}\right)}{n}\right)$ is the same for all members.

The expected utility achieved by member $i$ in the agreement (see equation 1) is then simply written as:

$$
u_{i}(\vec{a}, q, n)=\mathbb{E}\left[U\left(\widetilde{W}_{i}(\vec{a}, q, n)\right)\right]-C\left(a_{i}\right)
$$

We now write $v(a, q, n) \equiv u_{i}([a, \ldots, a], q, n)$ as the utility obtained by each individual when all participants play the same action $a$, (this utility does not depend on $i$, since all the members face 
the same individual risk):

$$
\begin{aligned}
v(a, q, n) & \equiv u_{i}([a, \ldots, a], q, n)=\mathbb{E}\left[U\left(\widetilde{W}_{i}(\vec{a}, q, n)\right)\right]-C(a) \\
& =\mathbb{E}\left[U\left(w-\left((1-q) \tilde{\delta}_{i}+\frac{q}{n} \sum_{j=1}^{n} \tilde{\delta}_{j}\right)\right) L(a)\right]-C(a)
\end{aligned}
$$

Thanks to the assumptions on $U(\cdot), C(\cdot)$ and $L(\cdot)$, function $v$ is strictly concave in $a$ and $q$.

In the optimal agreement, the action and the coverage are chosen simultaneously by the pool. In particular, the optimal collective action $a^{C}$ and the optimal coverage $q^{* C}$ solve the two first order conditions. The one for $a$ is:

$$
\frac{\partial v}{\partial a}=\left[-\mathbb{E}\left[U^{\prime}\left(\widetilde{W}_{i}\right) \widetilde{\delta}_{i}\right]\left(1-q+\frac{q}{n}\right)-\sum_{j \neq i} \mathbb{E}\left[U^{\prime}\left(\widetilde{W}_{i}\right) \widetilde{\delta}_{j}\right] \frac{q}{n}\right] L^{\prime}(a)-C^{\prime}(a)=0
$$

where the term $-\mathbb{E}\left[U^{\prime}\left(\widetilde{W}_{i}\right) \widetilde{\delta}_{i}\right]\left(1-q+\frac{q}{n}\right) L^{\prime}(a)$ indicates the total effect of the action chosen by individual $i$ on his/her utility from consumption, whereas the term $-\sum_{j \neq i} \mathbb{E}\left[U^{\prime}\left(\widetilde{W}_{i}\right) \widetilde{\delta}_{j}\right] \frac{q}{n} L^{\prime}(a)$ measures the effect of the action chosen by all the other members of the pool on the contribution paid by the same individual $i$. The first order condition for $q$ is:

$$
\frac{\partial v}{\partial q}=\mathbb{E}\left[U^{\prime}\left(\widetilde{W}_{i}\right)\left(\widetilde{\delta}_{i}-\frac{\sum_{j=1}^{n} \widetilde{\delta}_{j}}{n}\right)\right] L(a)=\frac{n-1}{n}\left[\mathbb{E}\left[U^{\prime}\left(\widetilde{W}_{i}\right) \widetilde{\delta}_{i}\right]-\mathbb{E}\left[U^{\prime}\left(\widetilde{W}_{i}\right) \widetilde{\delta}_{j}\right]\right] L(a)=0
$$

where the second equality comes from the fact that, because of i.i.d of the deltas: $\forall i, \forall k \neq i, \ell \neq$ $i, \mathbb{E}\left[U^{\prime}\left(\widetilde{W}_{i}\right) \widetilde{\delta}_{k}\right]=\mathbb{E}\left[U^{\prime}\left(\widetilde{W}_{i}\right) \widetilde{\delta}_{\ell}\right]$.

Using definition 11 we observe that, for $q=1, \widetilde{W}_{i}=w-\frac{\sum_{j=1}^{n} \tilde{\delta}_{j}}{n} L(a)=\widetilde{W}$ does not depend on $i$ Hence, for $q=1, \mathbb{E}\left[U^{\prime}\left(\widetilde{W}_{i}\right) \widetilde{\delta}_{i}\right]=\mathbb{E}\left[U^{\prime}\left(\widetilde{W}_{i}\right) \widetilde{\delta}_{j}\right]$.

Hence, $q^{* C}=1$ is the unique solution of 14 . 


\subsection{The first-best linear contract with fixed contribution}

We consider here the optimal linear contract with fixed contribution (premium) when the action can be directly controlled by the insurer. The individual receives $q L(a)$ when the loss occurs and, under fair pricing, the contribution equals $p q L(a)$. The expected utility becomes:

$$
\mathbb{E} U(w-[(1-q) \widetilde{\delta}+p q] L(a))-C(a)
$$

By maximizing the previous expected utility with respect to $q$, we find full coverage. Maximizing

with respect to the action and considering that $q^{F B}=1$, the action $a^{F B}$ is the implicit solution of:

$$
-p U^{\prime}(w-p L(a)) L^{\prime}(a)=C^{\prime}(a)
$$

The left-hand side of (16) indicates the marginal benefit while the right-hand side represents the marginal cost of the action. Importantly, the contribution $p q L(a)=p L(a)$ implies that individuals perfectly internalize the beneficial effect of their action on the contribution (see the term $U^{\prime}(w-$ $p L(a)))$. In particular, they take into account that a higher action, by decreasing the contribution, has a positive impact on marginal utility in both the possible states of nature. Marginal benefit is increasing in $p$ and in $-L^{\prime}(a)$, i.e. the efficiency of the action technology. Individuals' welfare is maximized and corresponds to:

$$
\mathbb{E} U^{F B}=U\left(w-p L\left(a^{F B}\right)\right)-C\left(a^{F B}\right)
$$

\subsection{Proof of Lemma 1}

We develop the proof in three steps: first we show existence, second we prove symmetry and uniqueness.

- Existence: since $u_{i}($.$) , as function of \vec{a}$, is continuous and concave and the set of actions is convex, a Nash equilibrium exists. 
- Best Response: for all $i \in\{1, \ldots, n\}$, consider for an individual $i$ the equation defining his/her best response:

$$
\begin{aligned}
& a_{i}=B R_{i}\left(\vec{a}_{-i}\right) \\
\Leftrightarrow & \mathbb{E}\left[U^{\prime}\left(\left(1-q+\frac{1}{n}\right)\left(w-\widetilde{\delta}_{i} L\left(a_{i}\right)\right)+q\left(w-\frac{\sum_{k \neq i} \widetilde{\delta}_{k} L\left(a_{j}\right)}{n}\right)\right) \widetilde{\delta}_{i}\right]\left(1-q+\frac{q}{n}\right)+\frac{C^{\prime}\left(a_{i}\right)}{L^{\prime}\left(a_{i}\right)}
\end{aligned}
$$

Or

$$
a_{i}=B R_{i}\left(\vec{a}_{-i}\right) \Leftrightarrow \mathbb{E}\left[U^{\prime}\left(\widetilde{W}_{i}(\vec{a})\right) \widetilde{\delta}_{i}\right]\left(1-q+\frac{q}{n}\right)+\frac{C^{\prime}\left(a_{i}\right)}{L^{\prime}\left(a_{i}\right)}=0, i=1, \ldots n
$$

Fix $i$ and $j$, and consider the partial function $f_{i}: a_{j} \rightarrow B R_{i}\left(a_{j}, \vec{a}_{-\{i, j\}}\right)$, where $\vec{a}_{-\{i, j\}}$ is given:

$$
\begin{aligned}
f_{i}^{\prime}\left(a_{j}\right) & =\frac{\mathbb{E}\left[U^{\prime \prime}\left(\widetilde{W}_{i}(\vec{a})\right) \widetilde{\delta}_{i} \widetilde{\delta}_{j}\right]\left(1-q+\frac{q}{n}\right) \frac{q}{n}}{\mathbb{E}\left[-U^{\prime \prime}\left(\widetilde{W}_{i}(\vec{a})\right) \widetilde{\delta}_{i}^{2}\right]\left(1-q+\frac{q}{n}\right)^{2}+\left(-\frac{C^{\prime}}{L^{\prime}}\right)^{\prime}\left(a_{i}\right)} \\
& =\frac{p^{2} \mathbb{E}\left[U^{\prime \prime}\left(\widetilde{W}_{i}(\vec{a})\right) / \widetilde{\delta}_{i} \widetilde{\delta}_{j}=1\right]\left(1-q+\frac{q}{n}\right) \frac{q}{n}}{p \mathbb{E}\left[-U^{\prime \prime}\left(\widetilde{W}_{i}(\vec{a}) / \widetilde{\delta}_{i}=1\right)\right]\left(1-q+\frac{q}{n}\right)^{2}+\left(-\frac{C^{\prime}}{L^{\prime}}\right)^{\prime}\left(a_{i}\right)}
\end{aligned}
$$

which is, given our assumptions on $C(\cdot), L(\cdot)$ and $U($.$) , negative. Actions are then strategic$ substitutes. Moreover we have:

$$
\begin{aligned}
\left|f_{i}^{\prime}\left(a_{j}\right)\right| & =\frac{p^{2} \mathbb{E}\left[-U^{\prime \prime}\left(\widetilde{W}_{i}(\vec{a})\right) / \widetilde{\delta}_{i} \widetilde{\delta}_{j}=1\right]\left(1-q+\frac{q}{n}\right) \frac{q}{n}}{p \mathbb{E}\left[-U^{\prime \prime}\left(\widetilde{W}_{i}(\vec{a}) / \widetilde{\delta}_{i}=1\right)\right]\left(1-q+\frac{q}{n}\right)^{2}+\left(-\frac{C^{\prime}}{L^{\prime}}\right)^{\prime}\left(a_{i}\right)} \\
& <\frac{p^{2} \mathbb{E}\left[-U^{\prime \prime}\left(\widetilde{W}_{i}(\vec{a})\right) / \widetilde{\delta}_{i} \widetilde{\delta}_{j}=1\right]\left(1-q+\frac{q}{n}\right) \frac{q}{n}}{p \mathbb{E}\left[-U^{\prime \prime}\left(\widetilde{W}_{i}(\vec{a}) / \widetilde{\delta}_{i}=1\right)\right]\left(1-q+\frac{q}{n}\right)^{2}} \\
\left|f_{i}^{\prime}\left(a_{j}\right)\right|< & \frac{p \mathbb{E}\left[-U^{\prime \prime}\left(\widetilde{W}_{i}(\vec{a})\right) / \widetilde{\delta}_{i} \widetilde{\delta}_{j}=1\right]\left(1-q+\frac{q}{n}\right) \frac{q}{n}}{\mathbb{E}\left[-p U^{\prime \prime}\left(\widetilde{W}_{i}(\vec{a}) / \widetilde{\delta}_{i} \widetilde{\delta}_{j}=1\right)-(1-p) U^{\prime \prime}\left(\widetilde{W}_{i}(\vec{a}) / \widetilde{\delta}_{i}=1, \widetilde{\delta}_{j}=0\right)\right]\left(1-q+\frac{q}{n}\right)^{2}} \\
\left|f_{i}^{\prime}\left(a_{j}\right)\right| & <\frac{p \mathbb{E}\left[-U^{\prime \prime}\left(\widetilde{W}_{i}(\vec{a})\right) / \widetilde{\delta}_{i} \widetilde{\delta}_{j}=1\right]\left(1-q+\frac{q}{n}\right) \frac{q}{n}}{p \mathbb{E}\left[-U^{\prime \prime}\left(\widetilde{W}_{i}(\vec{a}) / \widetilde{\delta}_{i} \widetilde{\delta}_{j}=1\right)\right]\left(1-q+\frac{q}{n}\right)^{2}}=\frac{q}{\left(1-q+\frac{q}{n}\right)} \leq 1
\end{aligned}
$$

So that $0 \geq f_{i}^{\prime}\left(a_{j}\right)>-1$. 
- Symmetry : suppose that there exists a Nash equilibrium for which there are two individuals $i$ and $j$ such that $a^{\prime}=a_{i}^{N} \neq a_{j}^{N}=a^{\prime \prime}$. Then, by symmetry, $a_{i}=a^{\prime \prime}, a_{j}=a^{\prime}$ and $a_{k}=a_{k}^{N}$ is also a Nash equilibrium. That means that $a^{\prime}=f_{i}\left(a^{\prime \prime}\right)$ and $a^{\prime}=f_{i}\left(a^{\prime \prime}\right)$, so that $\frac{f_{i}\left(a^{\prime}\right)-f_{i}\left(a^{\prime \prime}\right)}{a^{\prime}-a^{\prime \prime}}=-1$. But by Rolles theorem, this implies that there exists $a$ such that $f_{i}^{\prime}(a)=-1$, which is impossible.

- Now, the condition defining the symmetric Nash equilibrium $a^{N}(q, n)$ reduces to :

$$
E\left[U^{\prime}\left(\widetilde{W}_{i}\right) \widetilde{\delta}_{i}\right]\left(1-q+\frac{q}{n}\right)+\frac{C^{\prime}(a)}{L^{\prime}(a)}=0
$$

Once again, given our assumptions on $C(\cdot), L(\cdot)$ and $U(\cdot)$, this equation has a unique solution.

\subsection{Proof of Proposition 2}

Using the definition of $\widetilde{W}_{i}(\vec{a}, q, n)$ in 11 above (see Appendix 9.1), the expected utility achieved by member $i$ in the agreement, described by equation $(1)$ in the main text, can be rewritten in a compact form as:

$$
u_{i}(\vec{a}, q, n)=\mathbb{E}\left[U\left(\widetilde{W}_{i}(\vec{a}, q, n)\right)\right]-C\left(a_{i}\right)
$$

Recall that, in the first stage, individuals collectively choose the level of coverage $q$ and, in the second stage, each individual plays his/her best reply $a^{N}(q, n)$ to the actions chosen by the other members of the pool. From Lemma 9.3 the optimal action $a^{N}(q, n)$ is unique and symmetric and solves $\frac{\partial u_{i}}{\partial a_{i}}([a, a \ldots, a], q, n)=0$, or it satisfies equation $[19$.

Before focusing on the choice of $q$, we prove here that $\frac{\partial a^{N}}{\partial q}(q, n)<0$, or that the optimal action in the Nash equilibrium decreases in the amount of coverage provided by the pool. Denote $g(a, q, n)=\frac{\partial u_{i}}{\partial a_{i}}([a, a \ldots, a], q, n)$ expressed in $[19)$. By totally differentiating $g(a, q, n)$ with respect 
to $a$ and $q$ one finds:

$$
\begin{gathered}
\frac{\partial g}{\partial q}(a, q, n)=\mathbb{E}\left[U^{\prime}\left(\widetilde{W}_{i}\right) \widetilde{\delta}_{i}\right]\left(1-\frac{1}{n}\right) L^{\prime}(a) \\
+\mathbb{E}\left[U^{\prime \prime}\left(\widetilde{W}_{i}\right) \widetilde{\delta}_{i}\left(\widetilde{\delta}_{i} L(a)-\frac{\sum_{j=1}^{n} \tilde{\delta}_{j} L(a)}{n}\right)\right]\left(1-q+\frac{q}{n}\right) L^{\prime}(a)<0
\end{gathered}
$$

The first term is negative because $L^{\prime}\left(a_{i}\right)<0$, while the second term is negative because $U^{\prime \prime}\left(\widetilde{W}_{i}\right)<$ 0. Moreover,

$$
\begin{gathered}
\frac{\partial g}{\partial a}(a, q, n)=-\mathbb{E}\left[U^{\prime}\left(\widetilde{W}_{i}\right) \tilde{\delta}_{i}\right]\left(1-q+\frac{q}{n}\right) L^{\prime \prime}(a) \\
+\mathbb{E}\left[U^{\prime \prime}\left(\widetilde{W}_{i}\right) \tilde{\delta}_{i}\right]\left(1-q+\frac{q}{n}\right)\left(L^{\prime}(a)\right)^{2}-C^{\prime \prime}(a)<0
\end{gathered}
$$

where all terms are negative. Therefore, $\forall q \in[0,1]$,

$$
\frac{\partial a^{N}}{\partial q}(q, n)=-\frac{\partial g(a, q, n)}{\partial q} / \frac{\partial g(a, q, n)}{\partial a}<0
$$

Let us consider now the choice of $q$ in the first stage of the game and recall that $v(a, q, n)$ is the utility achieved by each individual when all participants play the same action $a$ (see also 12):

$$
v(a, q, n) \equiv u_{i}([a, \ldots, a], q, n)=\mathbb{E}\left[U\left(w-\left((1-q) \tilde{\delta}_{i}+\frac{q}{n} \sum_{j=1}^{n} \tilde{\delta}_{j}\right)\right) L(a)\right]-C(a)
$$

The action is now $a=a^{N}(q, n)$. Importantly, when collectively choosing $q$ in the first stage, the pool anticipates that all members will choose the action $a^{N}(q, n)$ without taking into account the positive impact that the action of other members exerts on the contribution to be paid. In different words and contrary to the case analyzed before (see Appendix 9.1), the action $a^{N}(q, n)$ is not the optimal one from the point of view of the pool in the first stage and, hence, the Envelope Theorem does not apply.

To see that, let us consider members' indirect utility $v\left(a^{N}(q, n), q, n\right)$. The optimal coverage 
here solves:

$$
\frac{\partial v}{\partial q}\left(a^{N}(q, n), q, n\right)=\frac{\partial v}{\partial q}\left(a^{N}(q, n), q, n\right)+\frac{\partial v}{\partial a^{N}}\left(a^{N}(q, n), q, n\right) \frac{\partial a^{N}}{\partial q}(q, n)=0
$$

where $\frac{\partial v\left(a^{N}(q, n), q, n\right)}{\partial a^{N}} \neq 0$. In fact, as mentioned before, $v\left(a^{N}(q, n), q, n\right) \neq y(q, n)=v\left(a^{C}(q, n), q, n\right)$ $=\max _{a} v(a, q, n)$.

From (14),

$$
\frac{\partial v}{\partial q}\left(a^{N}(q, n), q, n\right)=\frac{n-1}{n}\left(\mathbb{E}\left[U^{\prime}\left(\widetilde{W}_{i}\right) \widetilde{\delta}_{i}\right]-\mathbb{E}\left[U^{\prime}\left(\widetilde{W}_{i}\right) \widetilde{\delta}_{j}\right]\right) L\left(a^{N}\right)
$$

Moreover, when $q=1$, we showed in Appendix 9.1 that $\forall i, j, \widetilde{W}_{i}=\widetilde{W}_{j}$, so that :

$$
\frac{\partial v}{\partial q}\left(a^{N}(1, n), 1, n\right)=0
$$

In addition, using 13:

$$
\frac{\partial v}{\partial a}(a, q, n)=\left(-\mathbb{E}\left[U^{\prime}\left(\widetilde{W}_{i}\right) \widetilde{\delta}_{i}\right]\left(1-q+\frac{q}{n}\right)-\sum_{j \neq i} \mathbb{E}\left[U^{\prime}\left(\widetilde{W}_{i}\right) \widetilde{\delta}_{j}\right] \frac{q}{n}\right) L^{\prime}(a)-C^{\prime}(a) \neq 0
$$

In particular, for $q=1,24$ becomes:

$$
\begin{aligned}
\frac{\partial v}{\partial a}\left(a^{N}(1, n), 1, n\right) & =\left(-\frac{1}{n} \mathbb{E}\left[U^{\prime}\left(\widetilde{W}_{i}\right) \widetilde{\delta}_{i}\right]-\frac{n-1}{n} \mathbb{E}\left[U^{\prime}\left(\widetilde{W}_{i}\right) \widetilde{\delta}_{j}\right]\right) L^{\prime}\left(a^{N}(1, n)\right)-C^{\prime}\left(a^{N}(1, n)\right) \\
& =-\mathbb{E}\left[U^{\prime}\left(\widetilde{W}_{i}\right) \widetilde{\delta}_{i}\right] L^{\prime}\left(a^{N}(1, n)\right)-C^{\prime}\left(a^{N}(1, n)\right)
\end{aligned}
$$

Using (13), action $a^{N}(1, n)$ is such that:

$$
-\frac{1}{n} E\left[U^{\prime}\left(\widetilde{W}_{i}\right) \widetilde{\delta}_{i}\right] L^{\prime}\left(a^{N}(1, n)\right)-C^{\prime}\left(a^{N}(1, n)\right)=0
$$


By substituting the previous expression in 25) we find:

$$
\frac{\partial v}{\partial a}\left(a^{N}(1, n), 1, n\right)=-\frac{n-1}{n} E\left[U^{\prime}\left(\widetilde{W}_{i}\right) \widetilde{\delta}_{i}\right] L^{\prime}\left(a^{N}(1, n)\right)>0
$$

Using (23), 26) and (20), we are now in the position to evaluate 22 when $q=1$ :

$$
\frac{\partial v}{\partial q}\left(a^{N}(1, n), 1, n\right)=\frac{\partial v}{\partial a}\left(a^{N}(1, n), 1, n\right) \frac{\partial a^{N}}{\partial q}(1, n)<0
$$

which proves that $q=1$ does not satisfies 22 . Moreover, 27) shows that the function $v\left(a^{N}(q, n), q, n\right)$ is decreasing in $q$ when $q=1$, which implies that the optimal coverage is $q^{* N}(n)<1$.

\subsection{The second-best linear contract with fixed contribution}

Consider now the optimal linear contact with fixed contribution (premium) when the action cannot be controlled. Since the action is not contractible and moral hazard has bite, we call this policy the second-best contract with fixed contribution.

A policyholder pays the premium $P$ and receives the reimbursement $q L(a)$ if the loss realizes. The individual's expected utility is:

$$
\mathbb{E} U\left(w-\left[(1-q) \widetilde{\delta}_{i}\right] L(a)-P\right)-C(a)
$$

The timing of actions is the following: first, the forprofit insurer offers the contract $(P, q)$; second, the individual accepts the contract and chooses the action level; finally the risk is realized.

Solving backward, the optimal choice of the action, given the contract $(P, q)$, is the solution of:

$$
-p(1-q) U^{\prime}(w-(1-q) L(a)-P) L^{\prime}(a)=C^{\prime}(a)
$$

Obviously, with $q=1$, the optimal action is zero because the action does not bring any benefit in this case. Hence, the insurer will never offer full insurance. By comparing $(16)$ and $(29)$ we observe that, under the second best policy, the policyholder does not internalize the positive impact that 
the action has on his premium. In particular, in the left-hand side of 29 ) only the beneficial effect of the action on the potential loss is taken into account.

In the first stage, the insurer maximizes the policyholder's utility (28) subject to the resources constraint $(P=p q L(a))$ and the individual's incentive constraint 29. As is well known, the optimal level of coverage $q$ is lower than 1 (partial coverage), which means that the usual trade-off between risk-sharing and incentives arises.

Note that the fair contribution $P=p q L(a)$ is coherent both with the case of a benevolent monopolistic insurer (i.e. a public/social insurance) and with the case of a large number of forprofit insurers in a competitive market.

\subsection{Proof of Proposition 3}

Since $q^{* C}=1$, the level of utility achieved in the optimal risk-sharing agreement is written

$$
u^{C}(n)=\max _{a} \mathbb{E}\left[U\left(w-\frac{\sum_{i=1}^{n} \widetilde{\delta}_{i}}{n} L(a)\right)\right]
$$

Now, as the $\tilde{\delta}_{i}$ are i.i.d. with finite expectation, we know that $w-\frac{\sum_{i=1}^{n} \widetilde{\delta}_{i}}{n} L(a)$ is a mean preserving spread of $w-\frac{\sum_{i=1}^{n+1} \widetilde{\delta}_{i}}{n+1} L(a)$ for all $a$ so that :

$$
\mathbb{E}\left[U\left(w-\frac{\sum_{i=1}^{n} \widetilde{\delta}_{i}}{n} L(a)\right)\right] \leq \mathbb{E}\left[U\left(w-\frac{\sum_{i=1}^{n+1} \widetilde{\delta}_{i}}{n+1} L(a)\right)\right] \quad \forall a
$$

Therefore:

$$
u^{C}(n)=\max _{a} \mathbb{E}\left[U\left(w-\frac{\sum_{i=1}^{n} \widetilde{\delta}_{i}}{n} L(a)\right)\right] \leq u^{C}(n+1)=\max _{a} \mathbb{E}\left[U\left(w-\frac{\sum_{i=1}^{n+1} \widetilde{\delta}_{i}}{n+1} L(a)\right)\right]
$$

\subsection{Proof of Proposition 4}

We show below that the left-hand side (lhs) of 10 is equal to its right-hand side for $n=1$; whereas the lhs is lower than the right-hand side (rhs) for $n=+\infty$.

- For $n=1$, the sole individual will always choose the optimal action level. Thus, $u^{C}(n)=$ 
$\left.u^{D}(n)\right)=u^{P}(n)$ and condition 10 holds with equality.

- When $n \rightarrow+\infty$ the impact of one individual's action on the contribution is negligible so that deviation is always profitable. This can be seen by rewriting inequality 10 with $n \rightarrow+\infty$

$$
\begin{gathered}
\frac{1}{1-\sigma}\left[U\left(w-p L\left(a^{C}\right)\right)-C\left(a^{C}\right)\right] \geq \\
\frac{1}{1-\sigma(1-p)}\left[U\left(w-p L\left(a^{C}\right)\right)+\frac{p \sigma}{1-\sigma} u^{N}(0,1, \infty)\right]
\end{gathered}
$$

where

$$
u^{N}(0,1, \infty)=u^{P}(\infty)=U(w-p L(0))
$$

because incentives to free ride are the highest as possible when the size of the pool is infinite and the optimal action is $a^{N}(1, \infty)=0$, implying $C\left(a^{N}\right)=0$.

Rearranging we can write:

$$
C\left(a^{C}\right) \leq p \sigma\left[U[w-p L(0)]-\frac{U\left(w-p L\left(a^{C}\right)\right)}{1-\sigma(1-p)}\right]
$$

Since $\frac{1}{1-\sigma(1-p)}>1$ and $U(w-p L(0))<U\left(w-p L\left(a^{C}\right)\right)$, the rhs of the previous inequality is negative so that the latter is never satisfied. Thus, deviation is always profitable for $n \rightarrow+\infty$ and 10 does not hold.

From Proposition 3 we also know that $u^{C}(n)$ is monotonically increasing in $n$.

We conclude that either the rhs of 10 is always above its lhs and the optimal risk sharing agreement is never enforceable, or it exists a pool size $\hat{n}>1$ such that the rhs crosses from below the lhs in $\hat{n}$ and the rhs lies above the lhs for $n>\hat{n}$. Thus, a necessary condition for the optimal risk-sharing to be enforceable is that $n<\hat{n}$. The condition $n<\hat{n}$ is necessary but not sufficient because it is possible that the rhs crosses the lhs more than once so that subsets of $n$ belonging to the interval $(1, \hat{n})$ and such that the lhs lies above the rhs may in principle exist. However, in our simulations with CARA and CRRA utility functions, the lhs and rhs cross just once. 


\section{References}

[1] Alger, I. and W. Weibull (2010), "Kinship, Incentives, and Evolution", The American Economic Review 100, 1725-1758.

[2] Barigozzi F. (2004), "Reimbursing Preventive Care", Geneva Papers on Risk and Insurance Theory, 29(2), 165-186.

[3] Besley T. and M. Ghatak (2005), "Competition and Incentives with Motivated Agents", The American Economic Review 95(3), 616-636.

[4] Bramoullé, Y. and Kranton R. (2007), "Risk Sharing Across Communities", The American Economic Review 97(2), 70-74.

[5] Borch, K. (1962), "Equilibrium in a reinsurance market", Econometrica, 30, 424-444.

[6] Bourlès, R. and D. Henriet (2012), "Risk-sharing Contracts with Asymmetric Information", The Geneva Risk and Insurance Review, 37, 27-56.

[7] Caire, G. (2009), "La nouvelle logique d'action des mutuelles de santé : la mise en place de priorité santé mutualiste", Recma, Revue Internationale d'Economie Sociale, 311, 22-35.

[8] Doherty, N. and Dionne, D. (1993), "Insurance with undiversifiable risk: Contract structure and organizational form of insurance firms", Journal of Risk and Uncertainty, 6, 187-203.

[9] Ellis R. and Manning W. (2007), "Optimal health insurance for action and treatment", Journal of Health Economics, 26, 1128-1150

[10] Ehrlich, I. and Becker, G. (1972), "Market insurance, self-insurance and self-protection", Journal of Political Economy, 80, 623-648.

[11] Fafchamps, M. and Lund, S. (2003), "Risk Sharing Networks in Rural Philippines", Journal of Development Economics, 71(2), 261-287.

[12] Friedman J.W. (1971), "A Non-cooperative Equilibrium for Supergames", Review of Economic Studies, 38(113), 1-12. 
[13] Genicot, G. and D. Ray (2003), "Group Formation in Risk-Sharing Arrangements", Review of Economic Studies, 70, 87-113.

[14] Green, E. and R. Porter (1984), "Noncooperative Collusion under Imperfect Price Information", Econometrica, 52, 87-100.

[15] ICMIF (International Cooperative and Mutual Federation), Global Mutual Market Share report 2010, available at http://www.icmif.org/mms2010.

[16] Hendel, I. and A. Lizzeri, (2003). "The Role Of Commitment In Dynamic Contracts: Evidence From Life Insurance", The Quarterly Journal of Economics, 118(1), 299-327.

[17] Kerleau, M. (2009), "Le nouveau cadre institutionnel de la protection sociale complémentaire d'entreprise: quels enjeux pour les mutuelles santé?", Recma, Revue internationale d'économie sociale, $312,15-34$.

[18] Lee, W. and Ligon, J. (2001), "Moral Hazard in risk pooling arrangements", The Journal of Risk and Insurance, 68(1), 175-190.

[19] Ligon, J. and Thistle, P. (2005), "The formation of mutual insurers in markets with adverse selection", Journal of Business, 78, 529-555.

[20] Lippi Bruni, M., Rago S. and Ugolini C. (2012), "Il ruolo delle mutue sanitarie integrative", Società Il Mulino, ISBN 978-88-15-24100-9

[21] Mayers, D. and Smith, C. (1986), "Ownership Structure and Control: The Mutualization of Stock Life Insurance Companies", Journal of Financial Economics, 16, 73-98.

[22] Moreno I., Velazquez F. and Watt R. (2006), "Can Bonus-Malus Alleviate Insurance Fraud?", The Journal of Risk and Insurance, 73(1), 123-151.

[23] Neyman A. (1999), "Cooperation in Repeated Games When The Number of Stages is not commonly known", Econometrica, 67, 45-64. 
[24] Olson, Mancur (1965), The Logic of Collective Action: Public Goods and the Theory of Groups (Revised ed.). Harvard University Press.

[25] Ostrom, E. (2000), "Collective Action and the Evolution of Social Norms", Journal of Economic Perspectives 14(3), 137-158.

[26] Picard, P. (2014), "Participating Insurance contracts and the Rothschild-Stiglitz Equilibrium Puzzle", Geneva Risk and Insurance Review, 39, 153-175.

[27] Roth, A.E. and J.K. Murnighan (1978), "Equilibrium Behavior and Repeated Play of the Prisoner's Dilemma", Journal of Mathematical Psychology, 17(2), 189-198.

[28] Smith, B. and Stutzer, M. (1990)," Adverse Selection, aggregate uncertainty and the role for mutual insurance contracts", Journal of Business, 63, 493-510.

[29] Udry, C. (1995). "Risk and Saving in Northern Nigeria", American Economic Review, 85(5), $1287-1300$. 
Expected utility of the

representative individual

Optimal risk-sharing agreement

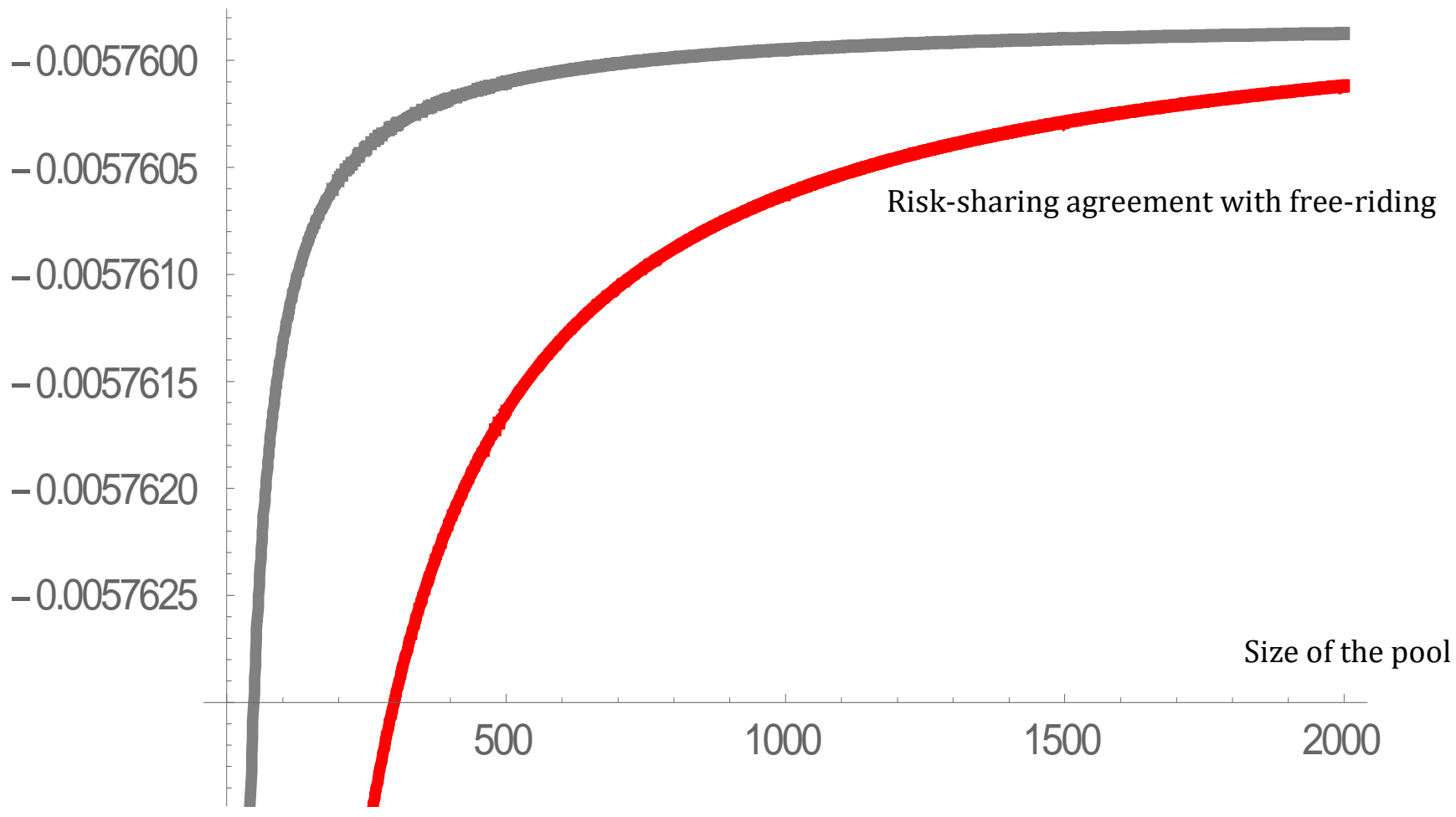

Figure 1. In the simulations with CARA and CRRA functions, the risk-sharing agreement with free-riding is monotonically increasing in the size of the pool. 
Expected utility of the

representative individual

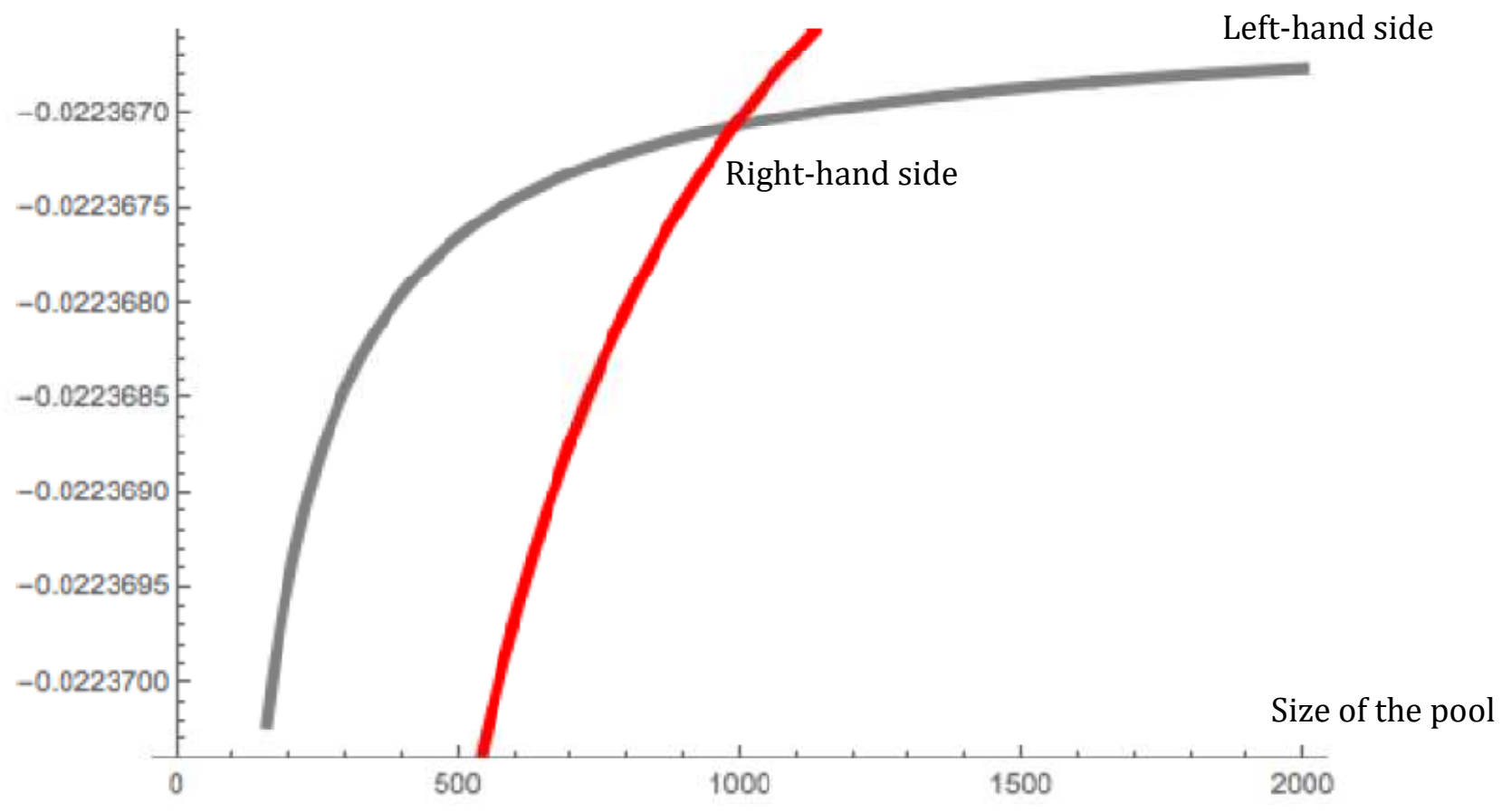

Figure 2. In the simulations with CARA utility function the curve which depicts the left-hand side of equation (10) lies above the curve representing its right-hand side for $\hat{n} \leq 1000$. 
Expected utility of the

representative individual

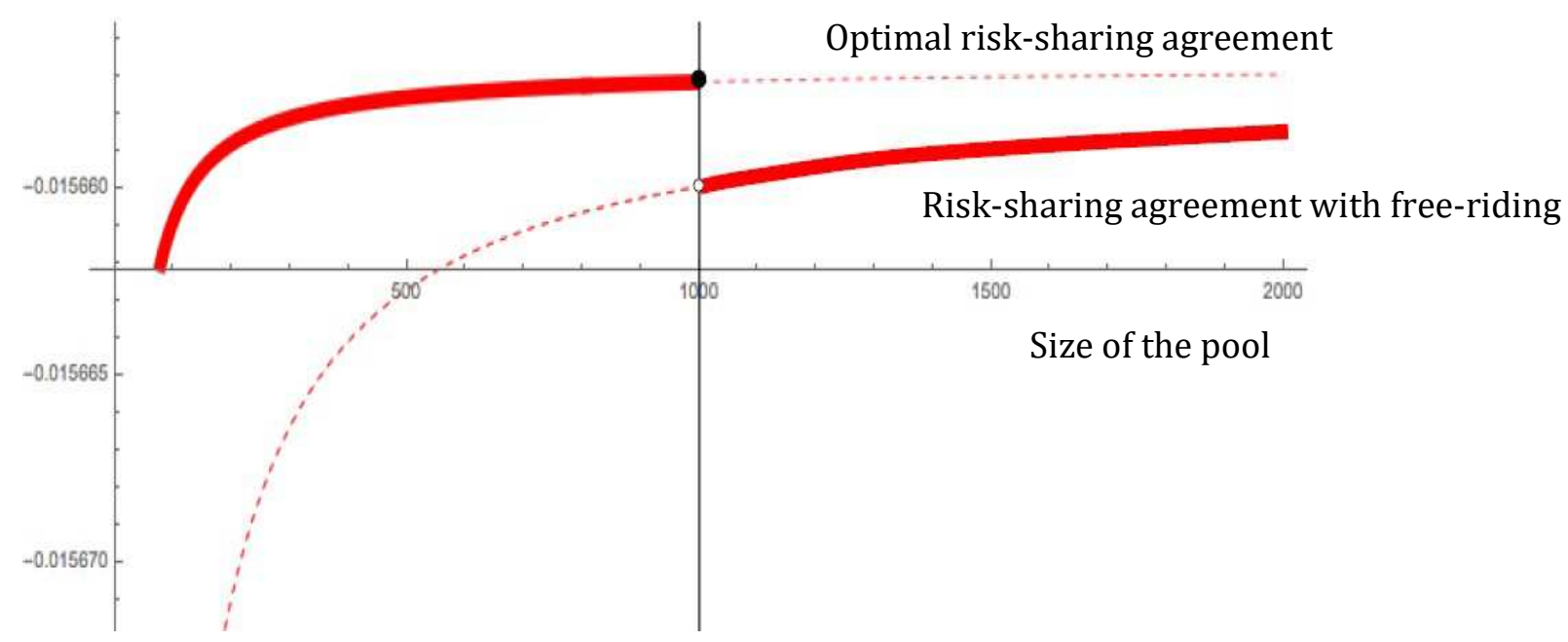

Figure 3. Simulations with CARA utility function. Solid lines describe the whole equilibrium schedule as a function of the size of the pool in the case where the optimal risk-sharing can be sustained as equilibrium for $\hat{n} \leq 1000$. 
Expected utility of the

representative individual

First-best policy with fixed premium

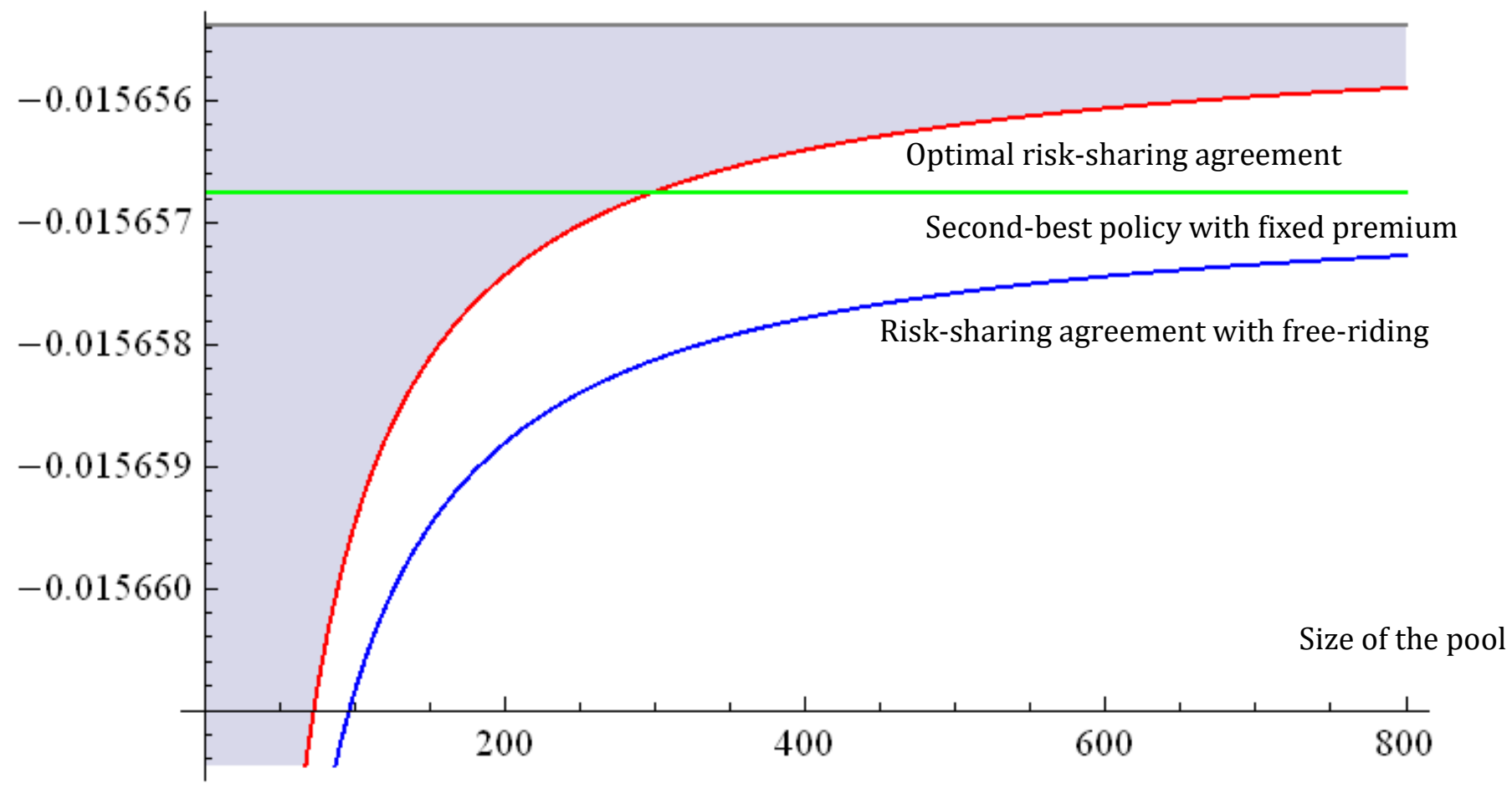

Figure 4. Members' welfare with the first-best policy with fixed premium, with the second-best policy with fixed premium, and in the risk-sharing agreement with and without free-riding using CARA utility functions. 